\title{
Large-eddy simulation of large-scale structures in long channel flow
}

\author{
D. CHUNG广 AND B. J. MCKEON \\ Graduate Aerospace Laboratories, California Institute of Technology, Pasadena, CA 91125, USA
}

(Received 22 November 2009; revised 27 May 2010; accepted 27 May 2010; first published online 23 August 2010)

We investigate statistics of large-scale structures from large-eddy simulation (LES) of turbulent channel flow at friction Reynolds numbers $R e_{\tau}=2 \mathrm{~K}$ and $200 \mathrm{~K}$ (where $\mathrm{K}$ denotes 1000). In order to capture the behaviour of large-scale structures properly, the channel length is chosen to be 96 times the channel half-height. In agreement with experiments, these large-scale structures are found to give rise to an apparent amplitude modulation of the underlying small-scale fluctuations. This effect is explained in terms of the phase relationship between the large- and small-scale activity. The shape of the dominant large-scale structure is investigated by conditional averages based on the large-scale velocity, determined using a filter width equal to the channel half-height. The conditioned field demonstrates coherence on a scale of several times the filter width, and the small-scale-large-scale relative phase difference increases away from the wall, passing through $\pi / 2$ in the overlap region of the mean velocity before approaching $\pi$ further from the wall. We also found that, near the wall, the convection velocity of the large scales departs slightly, but unequivocally, from the mean velocity.

Key words: turbulent boundary layers, turbulence simulation

\section{Introduction}

Recent studies (Kim \& Adrian 1999; Morrison et al. 2004; Guala, Hommema \& Adrian 2006; Hutchins \& Marusic 2007a, b; Monty et al. 2007; Mathis, Hutchins \& Marusic 2009a) have confirmed earlier observations (Favre, Gaviglio \& Dumas 1967; Kovasznay, Kibens \& Blackwelder 1970) of very long large-scale structures in the wall region of boundary layers, channels and pipes. These structures are visually marked by streamwise-elongated, alternating low- and high-momentum, meandering narrow regions, with lengths exceeding $20 \delta$ (Monty et al. 2007; Hutchins \& Marusic 2007a), where $\delta$ is the boundary-layer thickness, channel half-height or pipe diameter. See Balakumar \& Adrian (2007), Monty et al. (2009) for a description of the differences between the characteristics of these large structures in the different canonical flows. The bursting period of these structures, $6 \delta / U$, where $U(z)$ is the mean velocity and $z$ is the height from the wall, was already noted some decades ago by Cantwell (1981), who was reviewing the data of Rao, Narasimha \& Narayanan (1971). The dynamical significance of these large-scale structures can be seen in a scale decomposition of relative energy content, as measured by the pre-multiplied one-dimensional longitudinal spectrum $\kappa_{x} E_{u u}$ plotted against the $\log$ streamwise wavelength, $\log \lambda_{x}$, where $\lambda_{x}=2 \pi / \kappa_{x}$ (equal area under the curve implies equal energy contribution).

$\dagger$ Email address for correspondence: dchung@ caltech.edu 
For a boundary layer at friction Reynolds number $R e_{\tau}=7.3 \mathrm{~K}$ (where $\mathrm{K}$ denotes 1000 , Hutchins \& Marusic 2007b), the signature of these structures is related to the outer peak in $\kappa_{x} E_{u u}$ found at $\left(z / \delta, \lambda_{x} / \delta\right)=(0.06,6)$. Pipe flow studies indicate that this peak is located near $\lambda_{x} / \delta=12-14$ (Kim \& Adrian 1999) or $\lambda_{x} / \delta=10$ (Morrison et al. 2004). It has been proposed that the wall-normal location of this peak is located at the middle of the $\log$ layer in the boundary layer, $z^{+} \propto R e_{\tau}^{1 / 2}$ or $R e_{\tau}^{3 / 4}$ (Mathis et al. 2009a) (the choice of scaling depends on whether the lower limit of the log law is Reynoldsnumber-dependent) or $z^{+} \propto R e_{\tau}^{4 / 5}$ (McKeon \& Sharma 2010), where the superscript + indicates scaling in wall units: the friction velocity $u_{\tau}$ and kinematic viscosity $v$. However, the scaling remains somewhat ambiguous.

The large-scale structures were found (e.g. Bandyopadhyay \& Hussain 1984; Mathis et al. 2009a) to modulate the amplitudes of superimposed small-scale fluctuations. To test this idea, these authors first split the streamwise velocity into large- and small-scale components via a temporal filter at frequency $f=U(z) / \delta$, and then used either a filtered and rectified small-scale signal or the Hilbert transform to determine the envelope for the small-scale fluctuations, finally forming the correlation coefficient between the large-scale fluctuations and the low-pass-filtered envelope of the smallscale fluctuations. They found that, near the wall, large-scale high-speed regions carry intense superimposed small-scale fluctuations, but this correlation is reversed above a height that decreases in outer units with $R e_{\tau}$. We shall attempt to reproduce these features in this paper.

The footprint of structures centred far from the wall provides an obvious challenge in terms of determining appropriate convection velocities across the range of turbulent scales, with particular importance for obtaining the correct wavenumber spectra from temporal frequency spectra obtained by, for example, hot-wire anemometry. It has been known for some time that convection velocities deviate from the local mean in the near-wall region (e.g. Krogstad, Kaspersen \& Rimestad 1998). The common practice is to use Taylor's frozen-turbulence hypothesis to map from the frequency to the wavenumber domain, that is, to use the assumption that all structures at a given wall distance $z$ convect at the same scale-independent mean velocity $U(z)$. It was shown by a particle image velocimetry (PIV) experiment (Dennis \& Nickels 2008) that this is indeed good approximation at $z / \delta=0.16$ for a $R e_{\theta}=4.7 \mathrm{~K}$ boundary layer, at least for scales smaller than their field of view, $3.2 \delta$ in space and $6.3 \delta / U$ in time. However, note that this wall-normal distance is sufficiently far from the wall that it is beyond the large-scale energy peak, such that any convection velocity questions are likely to be insignificant because of the low shear in the outer region. With a field of view larger than $20 \delta \times 20 \delta / U$ and height down to $z / \delta=0.049$, we revisit the question of whether the footprint of the large-scale structures, having centres further from the wall, still convect at the local mean velocity near the wall.

To properly assess the dynamics of these long structures, reported to reach up to $25 \delta$ (Monty et al. 2007), we use large-eddy simulation (LES) coupled with a wall model (Chung \& Pullin 2009). This investigation is ideally suited to the present wall-modelled LES since its cost depends only on the number of "large eddies', which, for a channel, is Reynolds-number-independent. In contrast, the fully resolved direct numerical simulation (DNS) is prohibitively expensive. For reference, the most ambitious DNS of a channel flow to date is the $R e_{\tau}=2 \mathrm{~K}$ simulation (Hoyas \& Jiménez 2006) in an $L_{x} / \delta=8 \pi \approx 25$ channel, where $L_{x}$ is the streamwise length; a DNS investigation at higher $R e_{\tau}$ and larger $L_{x}$ of these largescale structures is not yet possible. Of course, the use of LES comes at the cost of subgrid-scale (SGS) modelling, wall modelling and numerical errors, but LES 


\begin{tabular}{lrcccllclllr}
\hline Run & $R_{\tau}$ & $L_{x} / \delta$ & $L_{y} / \delta$ & $h_{0}^{+}$ & $\Delta_{x} / \delta$ & $\Delta_{t} u_{\tau} / \delta$ & $N_{x}$ & $N_{y}$ & $N_{z}$ & $N_{t}$ & $T U_{c} / L_{x}$ \\
G1 & $2 \mathrm{~K}$ & 95 & 7.9 & 15 & 0.17 & 0.006 & 576 & 48 & 48 & 72000 & 110 \\
H3 & $200 \mathrm{~K}$ & 96 & 8.0 & 750 & 0.083 & 0.002 & 1152 & 96 & 96 & 15800 & 12 \\
G1b & $2 \mathrm{~K}$ & 95 & 7.9 & 15 & 0.17 & 0.006 & 576 & 48 & 48 & 22000 & 34
\end{tabular}

TABLE 1. LES parameters for long channel flows. Channel-transit times based on data-recording period $T$ and centreline velocity $U_{c}$.

is much faster (hours for simulation, minutes for post-processing) than DNS and experiments; we hope that a controlled application of the present LES combined with experience in the subject may shed some light on the physics of these large-scale structures.

Details of the simulations are given in $\S 2$ and discussion of observations are found in $\S 3$ before the conclusions in $\S 4$.

\section{Simulation details}

In the present paper, $x, y$ and $z$, respectively, denote the streamwise, spanwise and wall-normal directions; the velocity components, $u, v$ and $w$, are defined accordingly. As full details of the LES, including the numerical method and SGS model, are given by Chung \& Pullin (2009), we only highlight the important points here. We solve the filtered Navier-Stokes equations for the LES velocity field $\overline{\boldsymbol{u}}$ using the stretchedspiral vortex SGS model (Misra \& Pullin 1997; Voelkl, Pullin \& Chan 2000). To circumvent the inhibitive cost of resolving the near-wall region (Chapman 1979), $z<h_{0}$, we use a wall model (Chung \& Pullin 2009) that supplies off-wall slip-velocity boundary conditions at $h_{0}$ to the interior LES, operating in $h_{0}<z<2 \delta-h_{0}$, where $z=0,2 \delta$ locates the walls. In this paper, we fix $h_{0}=0.18 \Delta_{z}$, and the slip velocity is calculated using a wall model comprising (i) an evolution equation for the wall shear stress derived from assuming local inner scaling for the streamwise momentum equation and (ii) an extended form of the stretched-vortex SGS model that provides a local log relation along with a dynamic estimate for the local von Kármán constant. The parameters for the three LES runs are given in table 1. The grid is uniform, $\Delta_{x}=\Delta_{y}=4 \Delta_{z}$, throughout the simulation domain. To capture the physics of long large-scale structures, we use long a channel, $L_{x} / \delta \approx 96$, and the statistics are taken over $T U_{c} / L_{x}$ channel-transit times.

It was shown (Chung \& Pullin 2009) that the LES-predicted statistics from the $R e_{\tau}=2 \mathrm{~K}$ case, including means, turbulent intensities and spectra, compare reasonably well with the DNS of Hoyas \& Jiménez (2006). We show the root mean square (r.m.s.) of the streamwise velocity fluctuations and the LES-resolved spectra in figure 1. The points in figure $1(a)$ correspond to actual discretization points. Even though the total (subgrid plus resolved) r.m.s. is within $90 \%$ of the DNS result, its spectra plotted in energy-content form overpredict the DNS spectra by about $20 \%$. As such, the results presented here should be viewed as approximate, despite capturing energy at significantly larger wavelengths. On the other hand, the physics reported here can be seen as robust features of wall turbulence if they are also observed elsewhere. As an upper-bound estimate for the present model error, consider an SGS model constructed from random white noise. The statistics from this model are then a kind of input-output analysis (Jovanović \& Bamieh 2005) of the filtered unsteady threedimensional Navier-Stokes equations. White-noise spectra notwithstanding, we now 

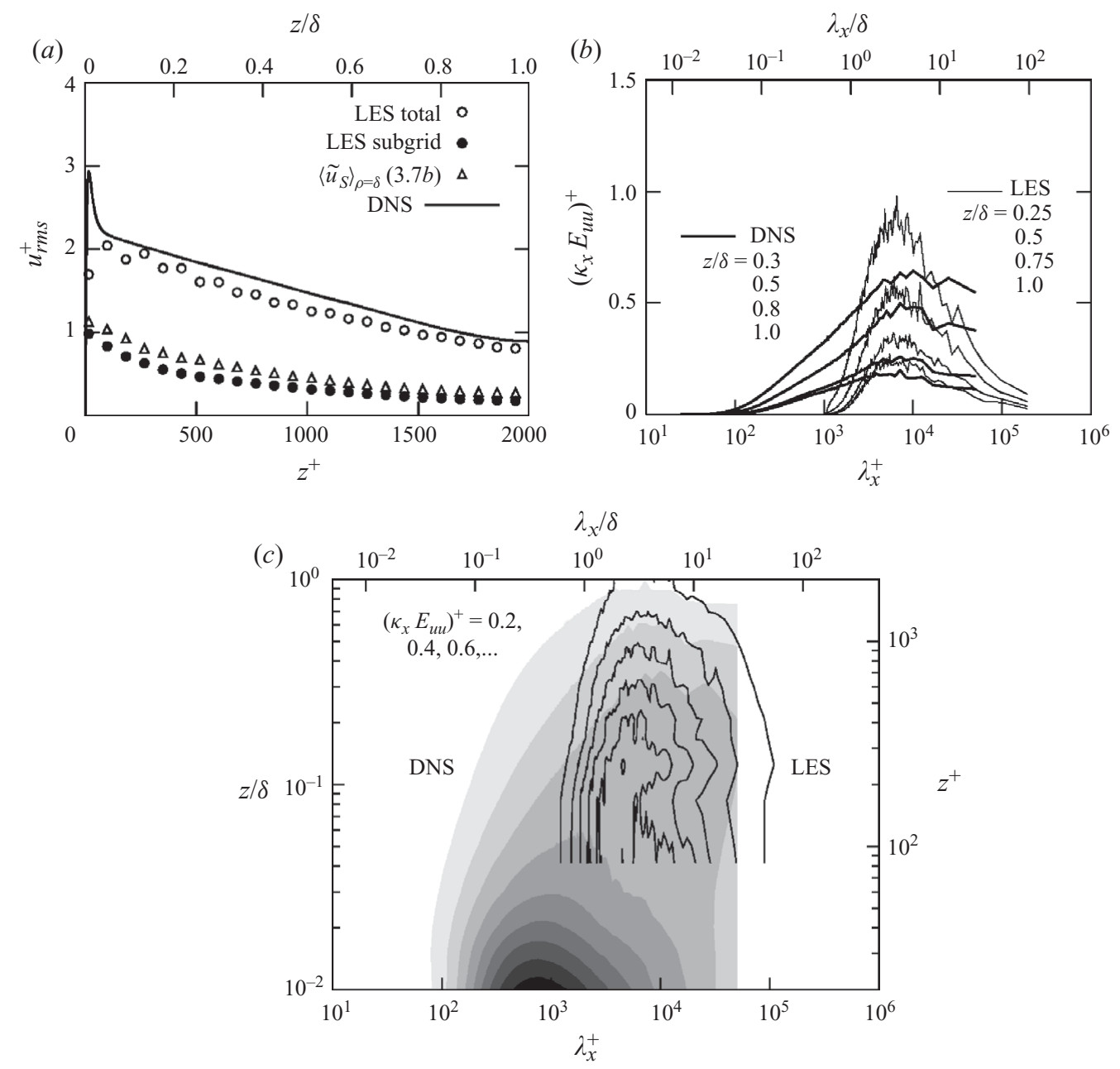

Figure 1. Comparison between DNS data of Hoyas \& Jiménez (2006) and present $R e_{\tau}=2 \mathrm{~K}$ channel flow LES, run G1b (table 1).

know that this simple model is capable of capturing many aspects of the large-scale structures (McKeon \& Sharma 2010). Therefore, we expect the present results, from the more realistic SGS model, to be even better at capturing the dynamics of the large-scale structures.

In order to compute correlations based on temporal averaging, a numerical rake in runs $\mathrm{G} 1$ and $\mathrm{H} 3$, fixed in streamwise-spanwise location, is set up to record the LES velocity $\bar{u}$ and its modelled subgrid fluctuations $T_{x x}(\equiv \overline{u u}-\bar{u} \bar{u})$ at the wall-normal locations $z=n_{z} \Delta_{z}\left(n_{z}=0,1, \ldots, N_{z}\right)$ and time steps $t=n_{t} \Delta_{t}\left(n_{t}=0,1, \ldots, N_{t}-1\right)$. Analogous correlations based on spatial averages are also computed from these runs from a snapshot in time. The only difference between runs G1 and G1b is the recorded data. For the latter, the three-dimensional data set, $\bar{u}\left(n_{x} \Delta_{x}, y, n_{z} \Delta_{z}, n_{t} \Delta_{t}\right)$, is recorded at fixed $y$, for $n_{x}=0,1, \ldots, 575, n_{z}=0,1, \ldots, 48$ and $n_{t}=0,1, \ldots, 21999$, where $\Delta_{x} / \delta=0.17, \Delta_{z} / \delta=0.041$ and $\Delta_{t} u_{\tau} / \delta=0.006$. 


\section{Discussion of observations}

We discuss our observations of the LES velocity fields, with emphasis on the large scales.

\subsection{Convection velocities from spatio-temporal spectra}

We begin by using the spatio-temporal data available in this study to investigate the validity of Taylor's hypothesis. This involves comparing the mean velocity, $U$, with the scale-dependent convection velocity, $U_{c}\left(\kappa_{x}\right)$, since these are equal where Taylor's hypothesis is valid.

\subsubsection{Spatio-temporal spectrum}

Given the autocorrelation of the streamwise velocity fluctuations,

$$
R(\rho, \tau)=\left\langle u^{\prime}(x, t) u^{\prime}(x+\rho, t+\tau)\right\rangle,
$$

where $\rho$ is the streamwise separation; $\tau$ is the time delay; \langle\rangle is the ensemble average; and $u \equiv U+u^{\prime}$ such that $\left\langle u^{\prime}\right\rangle=0$, we define the spatio-temporal spectrum $\Psi\left(\kappa_{x}, \omega\right)$ as the Fourier transform of $R(\rho, \tau)$. That is, together they form the Fourier transform pair, given by

$$
\begin{aligned}
\Psi\left(\kappa_{x}, \omega\right) & =\frac{1}{(2 \pi)^{2}} \int_{-\infty}^{\infty} \int_{-\infty}^{\infty} R(\rho, \tau) \mathrm{e}^{-\mathrm{i}\left(\kappa_{x} \rho-\omega \tau\right)} \mathrm{d} \rho \mathrm{d} \tau, \\
R(\rho, \tau) & =\int_{-\infty}^{\infty} \int_{-\infty}^{\infty} \Psi\left(\kappa_{x}, \omega\right) \mathrm{e}^{\mathrm{i}\left(\kappa_{x} \rho-\omega \tau\right)} \mathrm{d} \kappa_{x} \mathrm{~d} \omega .
\end{aligned}
$$

When computing $\Psi$ from the present LES simulation, a normalized Hann window in time, $W(t)=\sqrt{2 / 3}\left[1-\cos \left(2 \pi t /\left(M_{t} \Delta_{t}\right)\right)\right]$, where $M_{t} \Delta_{t}$ is discrete Fourier transform (DFT) temporal window size, is applied to the LES-resolved velocity $\bar{u}$ before taking the DFT because the velocity is not periodic in time. The spectrum is computed from run G1b (table 1) $\left(N_{t}=22000\right.$ and $\left.M_{t}=576\right)$, averaged across $\left\lfloor N_{t} /\left(M_{t} / 2\right)-1\right\rfloor=75$ half-overlapping windows (realizations). No windowing is necessary in the periodic streamwise direction. Explicitly, $\Psi\left(\kappa_{x}, \omega\right)=\left\langle\widehat{u}\left(\kappa_{x}, \omega\right) \widehat{u}^{*}\left(\kappa_{x}, \omega\right) /\left(\Delta_{\kappa_{x}} \Delta_{\omega}\right)\right\rangle$, where

$$
\widehat{u}\left(\kappa_{x}, \omega\right)=\sum_{t} \sum_{x} W(t) \bar{u}(x, t) \mathrm{e}^{\mathrm{i}\left(\kappa_{x} x-\omega t\right)}
$$

$t=\left(0, \ldots, M_{t}-1\right) \Delta_{t} ; \quad x=\left(0, \ldots, N_{x}-1\right) \Delta_{x} ; \quad \kappa_{x}=\left(-N_{x} / 2, \ldots, N_{x} / 2-1\right) \Delta_{\kappa_{x}} ;$ $\omega=\left(-M_{t} / 2, \ldots, M_{t} / 2-1\right) \Delta_{\omega} ; \Delta_{\kappa_{x}}=2 \pi / L_{x} ; \Delta_{\omega}=2 \pi /\left(\Delta_{t} M_{t}\right) ;$ and \langle\rangle is the ensemble average over realizations. The wavenumber spectrum $\Theta\left(\kappa_{x}\right)$ and the frequency spectrum $\Phi(\omega)$ are both related to $\Psi$ via

$$
\Theta\left(\kappa_{x}\right)=\int_{-\infty}^{\infty} \Psi\left(\kappa_{x}, \omega\right) \mathrm{d} \omega=\frac{1}{2} E_{u u}\left(\kappa_{x}\right), \quad \Phi(\omega)=\int_{-\infty}^{\infty} \Psi\left(\kappa_{x}, \omega\right) \mathrm{d} \kappa_{x},
$$

whence the mean square of $u$ fluctuations can be recovered from

$$
\left\langle u^{\prime 2}\right\rangle=\int_{-\infty}^{\infty} \int_{-\infty}^{\infty} \Psi\left(\kappa_{x}, \omega\right) \mathrm{d} \kappa_{x} \mathrm{~d} \omega=\int_{-\infty}^{\infty} \Phi(\omega) \mathrm{d} \omega=\int_{-\infty}^{\infty} \Theta\left(\kappa_{x}\right) \mathrm{d} \kappa_{x} .
$$

These are even, $\Phi(\omega)=\Phi(-\omega)$ and $\Theta\left(\kappa_{x}\right)=\Theta\left(-\kappa_{x}\right)$.

\subsubsection{Scale-dependent convection velocity}

In this paper, the scale-dependent convection velocity, $U_{c}\left(\kappa_{x}\right)$, is defined in terms of a dispersion relation, $\omega_{c}\left(\kappa_{x}\right)$, which, in turn, is defined in terms of $\Theta\left(\kappa_{x}\right)$ and $\Phi(\omega)$ :

$$
U_{c}\left(\kappa_{x}\right) \equiv \omega_{c}\left(\kappa_{x}\right) / \kappa_{x}, \quad \text { where } \Theta\left(\kappa_{x}\right) \equiv \omega_{c}^{\prime}\left(\kappa_{x}\right) \Phi\left(\omega_{c}\left(\kappa_{x}\right)\right), \quad \omega_{c}^{\prime}\left(\kappa_{x}\right) \equiv \mathrm{d} \omega_{c} / \mathrm{d} \kappa_{x} ;
$$


see also (5.2) of Monty \& Chong (2009) and (6.4) of del Álamo \& Jiménez (2009). That is, $\omega_{c}\left(\kappa_{x}\right)$ is the frequency-to-wavenumber mapping such that the wavenumber spectrum $\Theta\left(\kappa_{x}\right)$ can be recovered from the frequency spectrum $\Phi(\omega)$. In practice, we use the computationally simpler, but analytically equivalent, form

$$
\omega_{c}\left(\kappa_{x}\right)=K_{t}^{-1}\left(K_{x}\left(\kappa_{x}\right)\right), \quad K_{x}\left(\kappa_{x}\right) \equiv \int_{\kappa_{x}}^{\infty} \Theta\left(\kappa_{x}^{\prime}\right) \mathrm{d} \kappa_{x}^{\prime}, \quad K_{t}(\omega) \equiv \int_{\omega}^{\infty} \Phi\left(\omega^{\prime}\right) \mathrm{d} \omega^{\prime},
$$

where $K_{t}$ is monotonic and $K_{x}(0)=K_{t}(0)=\left\langle u^{\prime 2}\right\rangle / 2$. In terms of the present LES fields, we first compute the spatio-temporal spectrum, $\Psi\left(\kappa_{x}, \omega\right)$, from a two-dimensional DFT, (3.4), and then compute $\Theta\left(\kappa_{x}\right)$ and $\Phi(\omega)$ from (3.5) and finally compute $K_{x}\left(\kappa_{x}\right)$ and $K_{t}(\omega)$ to obtain $\omega_{c}\left(\kappa_{x}\right)$. The SGS scales are not represented in this part of the study. As summarized by del Álamo \& Jiménez (2009), approximations to $\omega_{c}\left(\kappa_{x}\right)$ are often used in the literature when $\Psi\left(\kappa_{x}, \omega\right)$ is not available. For example, $\omega_{1}\left(\kappa_{x}\right)$ (Wills 1964) effectively searches for the maximum of $\Psi\left(\kappa_{x}, \omega\right)$ along $\omega$ at fixed $\kappa_{x}$,

$$
\left.\partial_{U} \Psi\left(\kappa_{x}, \kappa_{x} U\right)\right|_{U=U_{1}\left(\kappa_{x}\right)}=\left.\partial_{\omega} \Psi\left(\kappa_{x}, \omega\right)\right|_{\omega=\omega_{1}\left(\kappa_{x}\right)}=0 ;
$$

$\omega_{2}\left(\kappa_{x}\right)$, also computed by del Álamo \& Jiménez (2009), searches for the maximum of $\Psi\left(\kappa_{x}, \omega\right)$ along $\kappa_{x}$ at fixed $\omega$,

$$
\left.\partial_{\kappa_{x}} \Psi\left(\kappa_{x}, \omega\right)\right|_{\omega=\omega_{2}\left(\kappa_{x}\right)}=0 ;
$$

and the recently proposed $\omega_{3}\left(\kappa_{x}\right)$ (del Álamo \& Jiménez 2009) is the first moment of $\Psi\left(\kappa_{x}, \omega\right)$ at fixed $\kappa_{x}$,

$$
\omega_{3}\left(\kappa_{x}\right)=\int_{-\infty}^{\infty} \omega \Psi\left(\kappa_{x}, \omega\right) \mathrm{d} \omega / \int_{-\infty}^{\infty} \Psi\left(\kappa_{x}, \omega\right) \mathrm{d} \omega,
$$

and so on. None of these approximations satisfy (3.7) exactly, but these approach $\omega_{c}\left(\kappa_{x}\right)$ when the iso-contours of $\Psi$ are locally symmetric in $\kappa_{x}$ and $\omega / U_{c}\left(\kappa_{x}\right)$ about the line $\omega_{c}\left(\kappa_{x}\right)$ because, then, the first moment and the maximum of the distribution $\Psi\left(\kappa_{x}, \omega\right)$ at fixed $\kappa_{x}$ or $\omega$ trace out similar (but not identical) lines. Here, we observe that this is often the case (see figure 2), but with higher asymmetry nearer the wall. This may explain the higher discrepancy between $\omega_{1}\left(\kappa_{x}\right), \omega_{2}\left(\kappa_{x}\right)$ and $\omega_{3}\left(\kappa_{x}\right)$ as the wall is approached, as shown in figure 2 of del Álamo \& Jiménez (2009). Note that $U_{c}\left(\kappa_{x}\right)$ is not the convection velocity of individual eddies. To first order, $\omega_{c}\left(\kappa_{x}\right)$ describes the apparent passing frequency of the most energetic eddies with wavenumber $\kappa_{x}$, although this is an integral effect over the range of energetic spanwise wavenumbers.

\subsubsection{Taylor's frozen-turbulence hypothesis}

Under the assumption of Taylor's frozen-turbulence hypothesis, $U_{c}\left(\kappa_{x}\right)=U_{T}\left(\kappa_{x}\right)=U$ and $\omega_{c}\left(\kappa_{x}\right)=\omega_{T}\left(\kappa_{x}\right)=U \kappa_{x}$ so, from (3.7), $\Theta\left(\kappa_{x}\right)=U \Phi\left(U \kappa_{x}\right)$. The strict interpretation of Taylor's hypothesis that all eddies at a particular wall-normal location travel at only one velocity $U$ can be relaxed to account for energetic eddies which travel at $U \pm \Delta U$, provided $\Psi\left(\kappa_{x}, \omega\right)$ is symmetric with respect to the $\omega=U \kappa_{x}$ line, that is,

$$
\Psi\left(\kappa_{x}, \omega\right)=\Psi\left(\omega / U, U \kappa_{x}\right),
$$

because (3.12) then relates the two definitions in (3.5):

$\Theta\left(\kappa_{x}\right) \equiv \int_{-\infty}^{\infty} \Psi\left(\kappa_{x}, \omega\right) \mathrm{d} \omega=\int_{-\infty}^{\infty} \Psi\left(\omega / U, U \kappa_{x}\right) \mathrm{d} \omega=U \int_{-\infty}^{\infty} \Psi\left(\kappa_{x}^{\prime}, U \kappa_{x}\right) \mathrm{d} \kappa_{x}^{\prime} \equiv U \Phi\left(U \kappa_{x}\right)$. 

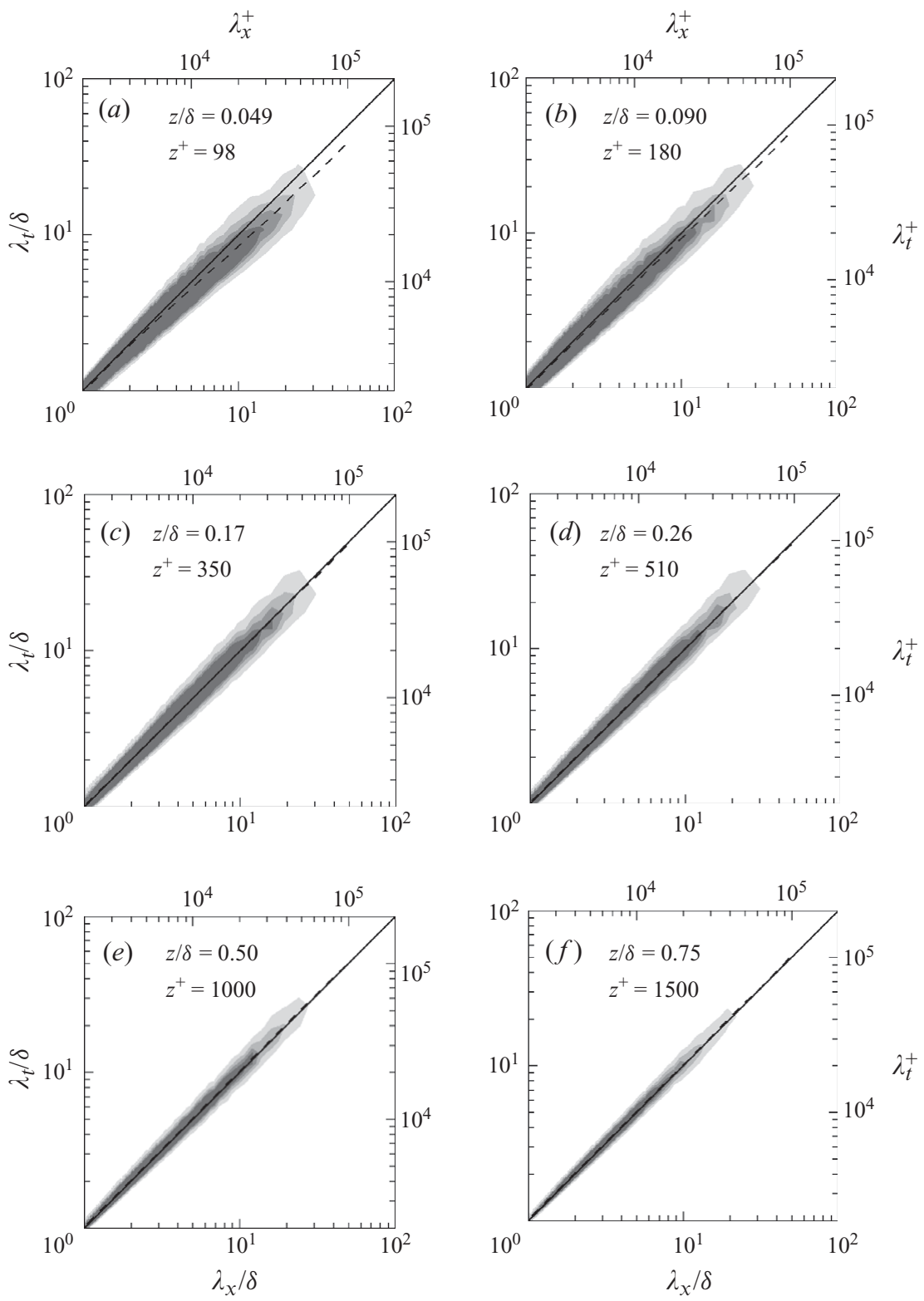

FIGURE 2. Pre-multiplied spatio-temporal spectra of streamwise velocity fluctuations, $\kappa_{x} \kappa_{t} \Psi / u_{\tau}^{2}=0.1,0.2,0.3,0.4$, at various heights from $R e_{\tau}=2 \mathrm{~K}$ channel flow LES, run G1b (table 1): ---- , dispersion relation computed from $\Psi, \lambda_{t}=\lambda_{x} \omega / \omega_{c} ;-$, Taylor's hypothesis $\lambda_{t}=\lambda_{x}$.

We plot in figure 2 contours of the pre-multiplied spectrum, $\kappa_{x} \kappa_{t} \Psi / u_{\tau}^{2}$ versus $\log \lambda_{x}$ and $\log \lambda_{t}$, where $\lambda_{x}=2 \pi / \kappa_{x}, \lambda_{t}=2 \pi / \kappa_{t}$ and $\kappa_{t}=\omega / U$. When Taylor's hypothesis is valid, contours of $\kappa_{x} \kappa_{t} \Psi / u_{\tau}^{2}$ should be symmetrical about the $\lambda_{t}=\lambda_{x}$ line, or, equivalently, $\omega_{c}\left(\kappa_{x}\right)=\omega_{T}\left(\kappa_{x}\right)$. Observe from figure 2 that Taylor's hypothesis is indeed a good approximation, except near the wall, $z / \delta=0.041$ and 0.083 , and for the large scales, $\lambda_{t} / \delta, \lambda_{x} / \delta>10$. Note that the LES formulation does not permit effective examination of smaller scales or locations closer to the wall. The dispersion relation 

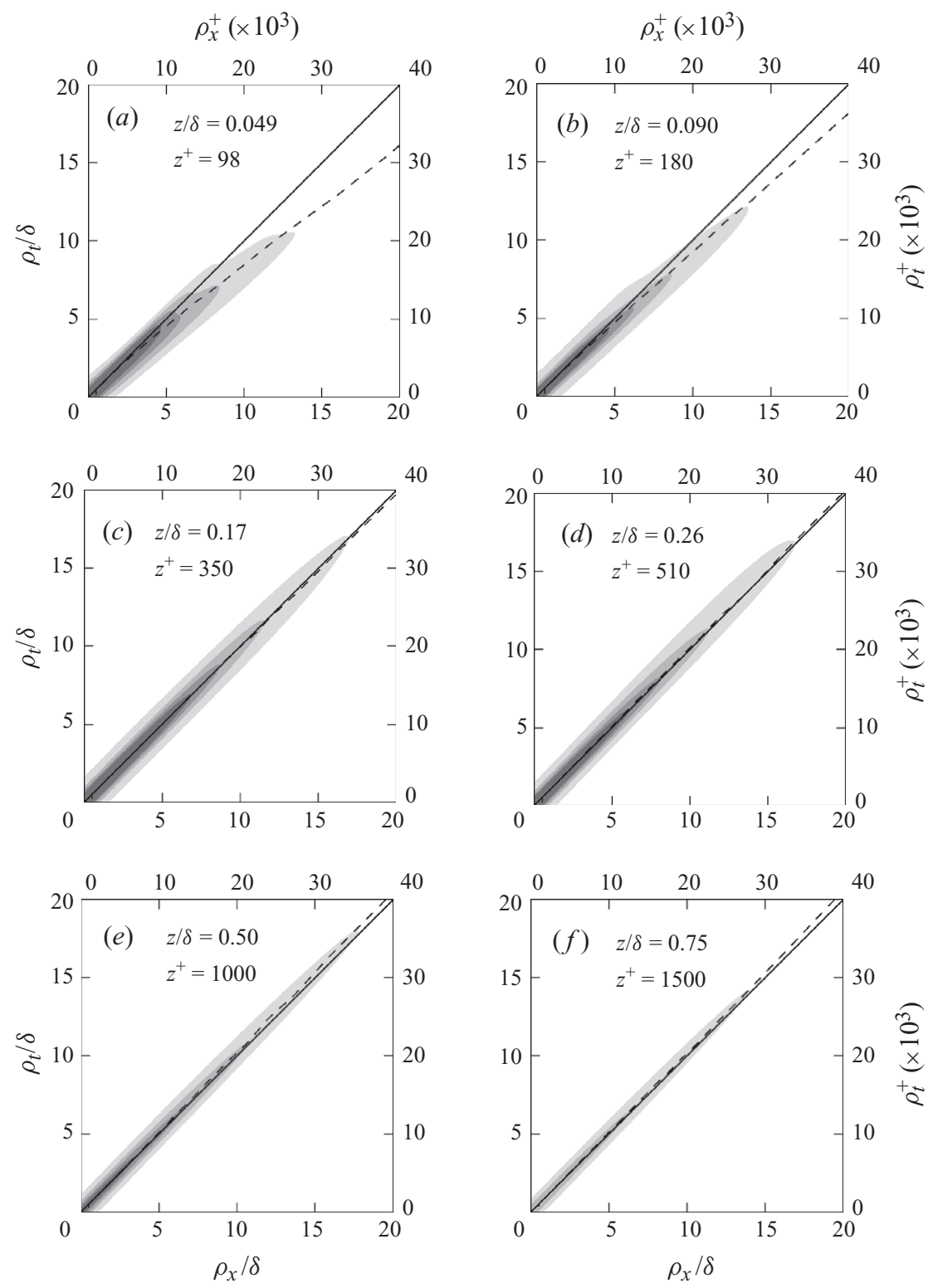

FIGURE 3. Spatio-temporal correlations of streamwise velocity fluctuations, $R / u_{\tau}^{2}=$ $0.3,0.6,0.9,1.2$, at various heights from $R e_{\tau}=2 \mathrm{~K}$ channel flow LES, run G1b (table 1): ---- , dispersion relation computed from $\Psi, \rho_{t}=\rho_{x} \omega / \omega_{c} ;-$, Taylor's hypothesis $\rho_{t}=\rho_{x}$.

line computed from $\Psi, \lambda_{t}=\lambda_{x} \omega / \omega_{c}$, appearing below (to the right) of Taylor's hypothesis, $\lambda_{t}=\lambda_{x} \omega / \omega_{T}=\lambda_{x} \cdot 1$, implies that the convection velocity $U_{c}\left(\kappa_{x}\right)=\omega_{c}\left(\kappa_{x}\right) / \kappa_{x}$ is larger than the mean velocity $U$.

Following Dennis \& Nickels (2008), we can also test the validity of Taylor's hypothesis in physical space by plotting the autocorrelation $R$ defined by (3.1). Taylor's hypothesis is valid, or more precisely there is a straightforward conversion from the temporal to the spatial domain, where the contours of $R$ are symmetrical about the $\rho_{x}=\rho_{t}$ line, where $\rho_{t}=\tau U$. Like figure $2, R$ in figure 3 shows an unequivocal departure 
from Taylor's hypothesis near the wall, $z / \delta=0.041$ and 0.083 and for the large scales, $\rho_{t} / \delta, \rho_{x} / \delta>10$. There also appears to be a slight discrepancy of the opposite sign for the large scales at $z / \delta=0.5$, which is more marked in the autocorrelation than our presentation of the spectrum. The boundary-layer PIV experiment performed by Dennis \& Nickels (2008) at $R e_{\theta}=4.7 \mathrm{~K}$ reported that Taylor's hypothesis is still valid at the height $z / \delta=0.16$ for the field of view $\rho / \delta<0.29 \mathrm{~m} / 0.09 \mathrm{~m}=3.2$ and $\tau U / \delta<1 \mathrm{~s} \times 0.57 \mathrm{~m} \mathrm{~s}^{-1} / 0.09 \mathrm{~m}=6.3$. This is consistent with the present LES data since at $z / \delta=0.17$ (figure $3 c$ ),$R$ is indeed symmetrical about the $\rho_{x}=\rho_{t}$ line, even up to very large scales $\rho_{t} / \delta, \rho_{x} / \delta=20$.

Consideration of spatio-temporal spectra permits some speculation about the convection velocities of the energetic structures and the error associated with identifying the footprint of eddies with a particular streamwise scale on the near-wall region from temporal data. Consistent with the recent DNS studies (del Álamo \& Jiménez 2009), the LES velocity fields unequivocally indicate that the most energetic large structures convect faster than the local mean velocity close to the wall, with the deviation growing close to the wall. Conversely, these large structures convect slower than the local mean velocity near the channel centre (figures $3 e$ and $3 f$ ). This suggests that these eddies are 'local' to a region in the overlap layer, in the sense that the mean velocity matches their convective velocity somewhere in the log region. We speculate that the location of this velocity matching corresponds to the location of the largescale streamwise energy peak, which is consistent with the approximate magnitude of the difference between $\omega / \omega_{T}$ and $\omega / \omega_{c}$ for large wavelength. This suggests that the departure from Taylor's hypothesis at the large scales and fixed wall units should strengthen with increasing Reynolds number due to the increasing shear near the wall. In other words, the departure from Taylor's hypothesis is most easily seen (i) at near-wall locations, (ii) at the large scales and (iii) for large Reynolds numbers. Our $R e_{\tau}=200 \mathrm{~K}$ data set has (ii) and (iii) but not (i). This is an area of current experimental study.

\subsection{Large-scale-small-scale interaction}

With the differences between the spatial and the temporal decompositions in mind, we now describe the correlation that characterizes the interaction between the large scales and the small scales. We first extract the large-scale fluctuations $u_{L}$ by applying a sliding-window top-hat time average, centred at $x$, to $u$ :

$$
u_{L}(x)=\frac{1}{\rho} \int_{x-\rho / 2}^{x+\rho / 2} u\left(x^{\prime}\right) \mathrm{d} x^{\prime},
$$

where $\rho$ is the width of the sliding window. A low-pass filter, (3.14), dampens fluctuations with frequencies higher than $1 / \rho$. In spectral space, (3.14) is equivalent to a multiplication by the filter $\sin \left(\rho \kappa_{x} / 2\right) /\left(\kappa_{x} / 2\right)$. For clarity, we have suppressed the $y$-, $z$ - and $t$-dependence of $u$ in this part of the discussion since these are held constant. The small-scale fluctuations are defined to be the remaining part of the motion, $u_{S}=u-u_{L}$. The small-scale intensity can be measured by its local r.m.s.,

$$
\widetilde{u}_{S}(x)=\left(\frac{1}{\rho} \int_{x-\rho / 2}^{x+\rho / 2} u_{S}^{2}\left(x^{\prime}\right) \mathrm{d} x^{\prime}\right)^{1 / 2} .
$$

Physically, $\widetilde{u}_{S}$ measures the local envelope or intensity of small-scale fluctuations. For example, if $u_{S}$ is normally distributed, $95 \%$ of its amplitude is estimated to lie within $2 \widetilde{u}_{S}$. If $u_{S}$ is not normal, $\widetilde{u}_{S}$ still measures the spread or envelope of $u_{S}$. In any case, 
$\widetilde{u}_{S}^{2}$ appears in the equations governing $u_{L}$, obtained by applying the filter (3.14) to the Navier-Stokes equations (see Reynolds \& Hussain (1972) for a related two-scale decomposition), which is another way to interpret $\widetilde{u}_{S}$. Note that equivalent approaches have been used by Bandyopadhyay \& Hussain (1984) and Guala, Metzger \& McKeon (2010) to obtain similar results in a range of flows including a laboratory turbulent boundary layer and the near-wall region of the near neutrally stable atmospheric surface layer, respectively.

An elegant alternative to obtain the envelope of $u_{S}$ is via the Hilbert transform (Mathis et al. 2009a), and this approach has led to a significant advance in understanding of the large-scale-small-scale interactions. However, it is harder to relate the results to the governing equations of turbulence. When calculating an r.m.s. defined locally, (3.15), one has to contend with the inevitability that large scales have been aliased into the small-scale signature. Perhaps a better alternative is to use a tapered window in (3.15), alleviating some but not all of the aliasing. We have tried this and found some minor changes, but the general picture is unaltered, and so we decided to keep the simple definition in (3.15). The Hilbert transform bypasses this aliasing difficulty at the enveloping stage, but the issue reappears when one filters the envelope signal. We note that even if a perfect decomposition can be found, nature herself does not permit it, that is, the two peaks in $\kappa_{x} E_{u u}$ (Hutchins \& Marusic 2007b) are never completely isolated, at least in Fourier space.

In terms of LES quantities, we can write (3.14) and (3.15) as

$$
\begin{aligned}
& u_{L}(x)=\frac{1}{\rho} \int_{x-\rho / 2}^{x+\rho / 2} \bar{u}\left(x^{\prime}\right) \mathrm{d} x^{\prime}, \\
& \widetilde{u}_{S}(x)=\left(\frac{1}{\rho} \int_{x-\rho / 2}^{x+\rho / 2}\left[u_{S}^{2}\left(x^{\prime}\right)+T_{x x}\left(x^{\prime}\right)\right] \mathrm{d} x^{\prime}\right)^{1 / 2},
\end{aligned}
$$

where $\bar{u}$ is the resolved velocity; $u_{S}=\bar{u}-u_{L}$; and $T_{x x}$ is the modelled subgrid fluctuations associated with scales smaller than the numerical discretization $\Delta_{x}$. Using (3.16), we now construct the normalized large-scale-small-scale correlation based on temporal filtering:

$$
R_{\rho}(z)=\frac{\left\langle\left(u_{L}-U\right)\left(\widetilde{u}_{S}-\left\langle\widetilde{u}_{S}\right\rangle\right)\right\rangle}{\left\langle\left(u_{L}-U\right)^{2}\right\rangle^{1 / 2}\left\langle\left(\widetilde{u}_{S}-\left\langle\widetilde{u}_{S}\right\rangle\right)^{2}\right\rangle^{1 / 2}},
$$

where the global or ensemble average is formally given by

$$
\langle\phi\rangle \equiv \lim _{L_{y}, L_{x} \rightarrow \infty} \frac{1}{L_{y} L_{x}} \int_{-L_{y} / 2}^{L_{y} / 2} \int_{-L_{x} / 2}^{L_{x} / 2} \phi\left(x^{\prime}, y^{\prime}\right) \mathrm{d} x^{\prime} \mathrm{d} y^{\prime} .
$$

Note that the visual envelope of $u_{S}$, e.g. $2 \widetilde{u}_{S}$, does not affect $R_{\rho}$ because the constant factor cancels out in the normalized correlation (3.17). In other words, $R_{\rho}$ does not contain amplitude information; it does, however, contain phase information, since it is the cosine of the angle (or phase) between $u_{L}-U$ and $\widetilde{u}_{S}-\left\langle\widetilde{u}_{S}\right\rangle$, using the inner product \langle\rangle . In practice, we obtain $R_{\rho}$ by replacing the integrals with sums and ensuring the averaging area $L_{x} L_{y}$ is much larger than the largest physical scale in the flow (see table 1). The spatial correlations are calculated from runs G1 and H3 at one snapshot in time. The temporal counterpart to (3.17), $R_{\tau}$, is defined analogously, with $t$ and $\tau$ respectively replacing $x$ and $\rho$, while holding other variables constant. 
(a) $z^{+}\left(\times 10^{2}\right)$

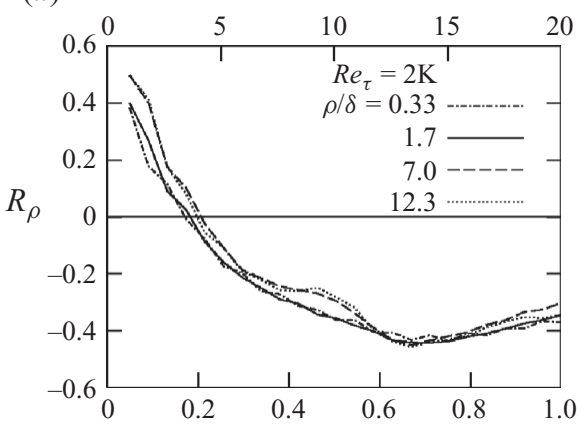

$z^{+}\left(\times 10^{4}\right)$

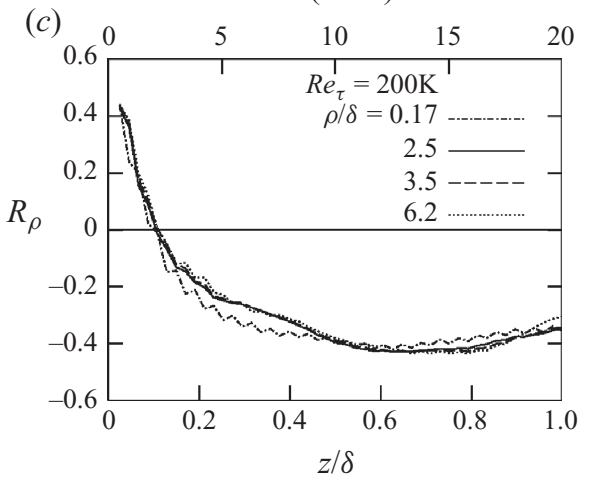

(b) $\quad z^{+}\left(\times 10^{2}\right)$

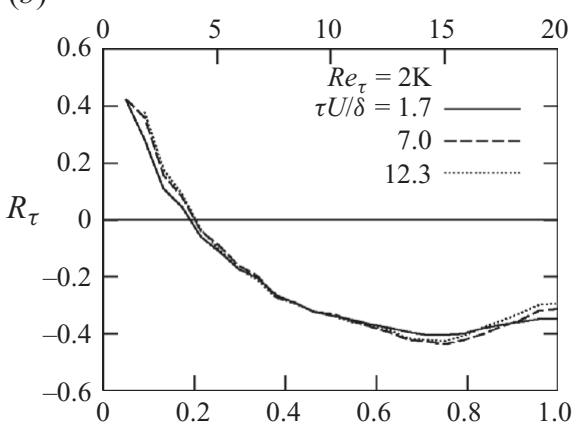

$z^{+}\left(\times 10^{4}\right)$

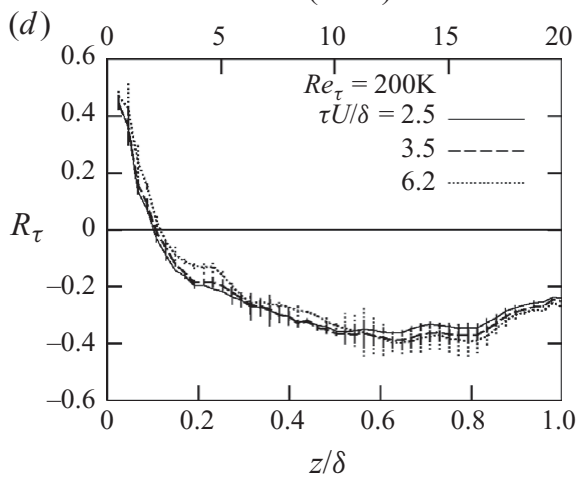

FiguRE 4. Profiles of large-scale-small-scale correlations, $R_{\tau}(b, d)$ and $R_{\rho}(a, c)$, defined by (3.17). Filter sizes and $R e_{\tau}$ inset. Error lines in (c) indicate convergence uncertainties from inadequate averaging period $T$. Data from channel flow LES, runs G1 and H3 (table 1).

For $R_{\tau}$, the wall-parallel plane average (3.18) is replaced by a long-time average over large $T$.

The physical meaning of the correlations $R=R_{\tau}$ or $R_{\rho}$ is as follows. If largescale higher-speed regions carry higher small-scale intensity (positively correlated, in phase), then $R \approx 1$. Similarly, if large-scale higher-speed regions carry lower small-scale intensity (negatively correlated, $\pi$ out of phase), then $R \approx-1 . R \approx 0$ can occur either if there is no correlation between the large and small scales, or if they are $\pi / 2$ out of phase, which is physically the more likely option given the strong correlation for small and large $z / \delta$, as already demonstrated by Bandyopadhyay \& Hussain (1984) and Mathis et al. (2009a). Although the r.m.s.-based correlation coefficient (3.17) is different from its Hilbert-transform-based counterpart in the boundary-layer study of Mathis et al. (2009a), we expect similar qualitative features if the large-scale-smallscale phase relationship is a universal aspect of wall-bounded flows, namely channels and boundary layers. We note that the accuracy of $R_{\rho}$ and $R_{\tau}$ largely depends on the accuracy of the modelled SGS stresses, which, for filter width $\rho=\delta$, constitutes approximately $80 \%$ of $\widetilde{u}_{S}$ (figure $1 a$ ).

Figure 4 compares the correlations based on spatial filtering, $R_{\rho}$, and correlations based on temporal filtering, $R_{\tau}$, for $R e_{\tau}=2 \mathrm{~K}$ and $R e_{\tau}=200 \mathrm{~K}$ and different values of $\rho$ and $\tau$. Observe that near the wall, $u_{L}$ and $\widetilde{u}_{S}$ are positively correlated, up to $R \approx 0.4$ 
(the maximum in the domain we resolve, although note that the maximum value likely increases closer to the wall), but above a certain crossing height, $z / \delta \approx 0.2$ for $R e_{\tau}=2 \mathrm{~K}$ and $z / \delta \approx 0.11$ for $R e_{\tau}=200 \mathrm{~K}$, they are negatively correlated, down to $R \approx-0.4$. The trend of decreasing crossing height with increasing Reynolds number is also reported by Mathis et al. (2009a) for the turbulent boundary layer, with $z / \delta \approx 0.07$ for $R e_{\tau}=2.8 \mathrm{~K}$ and $z / \delta \approx 0.03$ for $R e_{\tau}=19 \mathrm{~K}$. The correlations with $\rho / \delta=0.33\left(R e_{\tau}=2 \mathrm{~K}\right)$ and $\rho / \delta=0.17\left(R e_{\tau}=200 \mathrm{~K}\right)$ in figure $4(a, c)$ correspond to filtering with two LES grid points. That is, these largely represent the correlations between the resolved LES velocity field and SGS stresses. Note that the profiles of $R_{\rho}$ are largely determined by and highly sensitive to the resolved and subgrid-scale interaction. Therefore, it is possible that the exact details of subsequent analyses presented in the paper on the spatio-temporal relationship between the large and small scales could be highly sensitive to the exact SGS model used. We note, however, that the overall results show strong similarity with a range of experimental results across the full width of the channel, suggesting that the physics behind the subgrid-scale model is at least related to that in the fully resolved flow. In this paper, the correlations are largely independent of filter sizes $1.7<\tau U / \delta, \rho / \delta<12.3$, although the deviation between $R_{\rho}$ and $R_{\tau}$ with the larger filter sizes from the one with $\rho / \delta=1.7$ close to the wall is exacerbated in the spatial plots, as would be expected from the arguments concerning Taylor's hypothesis at the large scales in the preceding section. A small sensitivity to filter sizes is also reported by Mathis et al. (2009a). The kick-up of $R_{\rho}$ relative to $R_{\tau}$ for curves corresponding to $\rho / \delta=7.0,12.3$ in the vicinity of $z / \delta=0.45$ (figures $4 a$ and $4 b$ ) is presumably related to the convection velocity effect demonstrated in figure 3(e). Perhaps a precise quantitative comparison is impossible owing to the different envelope-extraction techniques and the different type of wallbounded flows. Note that a robust feature is that $R$ increases slightly at the centre of the channel, but remains negative (figure 4). This increase is also seen in boundary layers (Mathis et al. 2009a) (and this sensitivity appears to be slightly enhanced in the case of the spatial correlation), but the increase is from negative correlations to positive correlations, a feature possibly related to the intermittent boundary-layer thickness not present in channel flows, as confirmed by the recent comparison study by Mathis et al. (2009b).

Although the large-scale-small-scale interaction was recently (Mathis et al. 2009a) framed in terms of amplitude modulation, the results could also be discussed in terms of the relative phase between the large and small scales, as originally posed by Bandyopadhyay \& Hussain (1984) and implied by the formulation of (3.17). We examine this phase relationship in following section.

\subsection{Conditionally averaged large-scale velocities and small-scale intensities}

To gain some insight into the structure of the large-scale coherent regions and the phase relationship between $u_{L}$ and $\widetilde{u}_{S}$ shown in figure 4 , we now turn our attention to conditionally averaged $u_{L}$ and $\widetilde{u}_{S}$ fields computed with the streamwise filter window $\rho=\delta$. As seen in figure 4 and in Mathis et al. (2009a), this phase relationship is relatively unaffected by the choice of $\rho$.

Figure 5 shows ensemble averages, $\left\langle u_{L} \mid A_{1}\right\rangle(\Delta \boldsymbol{x})$ and $\left\langle\widetilde{u}_{S} \mid A_{1}\right\rangle(\Delta \boldsymbol{x})$, conditioned on the occurrence of a large-scale low-speed event at $z / \delta=0.090, A_{1}=u_{L}\left(\boldsymbol{x}_{\times}\right)-U<0$, where $\Delta \boldsymbol{x}=\boldsymbol{x}-\boldsymbol{x}_{\times}, \boldsymbol{x}_{\times}^{+}=(0,0,180) \Leftrightarrow \boldsymbol{x}_{\times} / \delta=(0,0,0.090)$, computed from a snapshot of the LES run G1b (table 1). The spanwise-wall-normal view (figure $5 a$ ) has previously been shown in figure 7(a) of Hutchins \& Marusic (2007b) with the choice $z_{\times}^{+}=150$ from the DNS data of del Álamo et al. (2004) at $R e_{\tau} \approx 1 \mathrm{~K}$. For reference, 

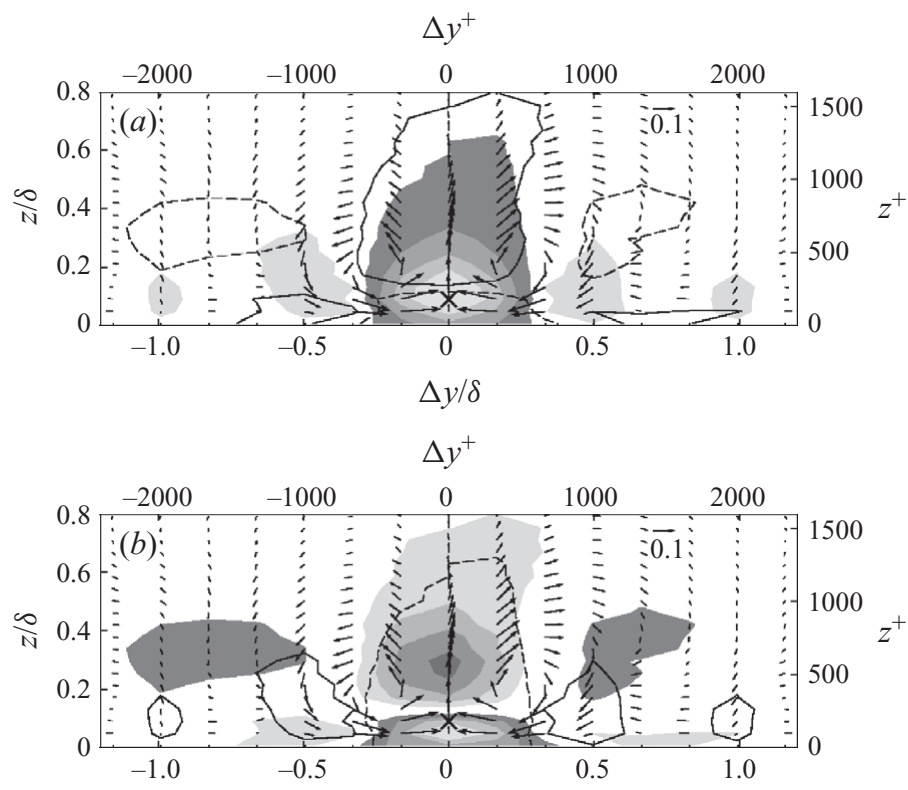

$\Delta y / \delta$
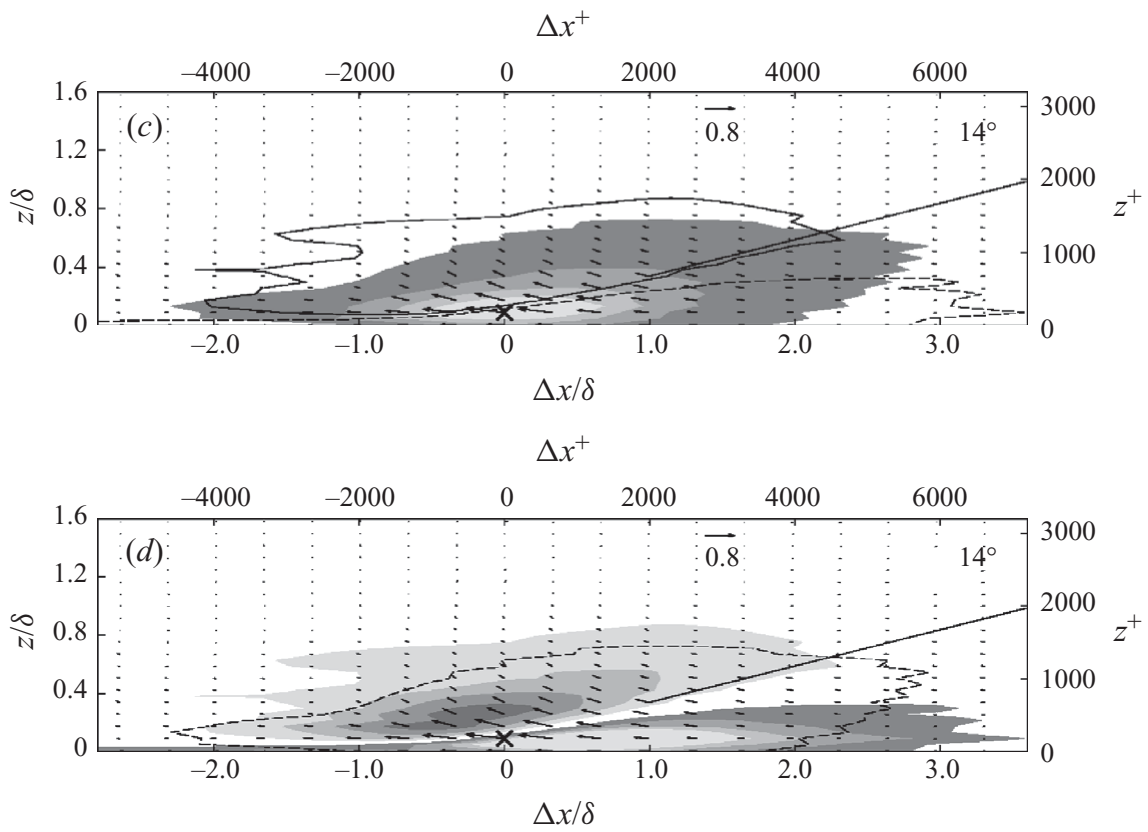

FIGURE 5. Conditionally averaged streamwise velocity fields in $(a, b)$ spanwise-wall-normal and $(c, d)$ streamwise-wall-normal planes, with $\left\langle\boldsymbol{u}_{L}^{+} \mid A_{1}\right\rangle(\Delta \boldsymbol{x})$ and $\left\langle\widetilde{u}_{S}^{+} \mid A_{1}\right\rangle(\Delta \boldsymbol{x})$, where $A_{1}$ is the low-speed event $u_{L}\left(\boldsymbol{x}_{\times}\right)-U<0$ with probability $P\left\{A_{1}\right\}=0.53$ at $\boldsymbol{x}_{\times} / \delta=(0,0,0.090)$ : $(a, c)$ filled contours of $\left|\left\langle u_{L}^{+} \mid A_{1}\right\rangle-U^{+}\right|=0.1,0.3,0.5,0.7$, line contours of $\left|\left\langle\widetilde{u}_{S}^{+} \mid A_{1}\right\rangle-\left\langle\widetilde{u}_{S}^{+}\right\rangle\right|=0.01$; and $(b, d)$ filled contours of $\left|\left\langle\widetilde{u}_{S}^{+} \mid A_{1}\right\rangle-\left\langle\widetilde{u}_{S}^{+}\right\rangle\right|=0.01,0.02,0.03,0.04$, line contours of $\left|\left\langle u_{L}^{+} \mid A_{1}\right\rangle-U^{+}\right|=0.1$. Darker to lighter shades and dashed lines for negative values. Vectors represent in-plane velocity components. Data from snapshot of $R e_{\tau}=2 \mathrm{~K}$ channel flow LES, run G1b (table 1). 
the vectors in figure $5(a, b)$ correspond to LES discretization points. The agreement between DNS and LES results is striking. The essential features of the DNS averages are also seen in the present LES averages, namely (i) a splatted low-speed region with minimum $\left\langle u_{L}^{+} \mid A_{1}\right\rangle \approx-0.8$ and width $\Delta y / \delta \approx 0.4$ centred on $\boldsymbol{x}_{\times}$flanked on both sides by weaker high-speed regions with maximum $u_{L}^{+} \approx 0.1$; and (ii) the in-plane large-scale swirl at $\Delta_{x} / \delta \approx \pm 0.2, z / \delta \approx 0.2$.

We report that $P\left\{A_{1}\right\}=0.53$, implying that nearly identical figures, but with signs reversed, are seen when we condition on the large-scale high-speed event $A_{1}^{\prime}=u_{L \times}-$ $U>0$ (the complement of $A_{1}$ ) because

$$
0=\langle\phi\rangle \equiv\left\langle\phi \mid A_{1}\right\rangle P\left\{A_{1}\right\}+\left\langle\phi \mid A_{1}^{\prime}\right\rangle P\left\{A_{1}^{\prime}\right\} \quad \Rightarrow \quad\left\langle\phi \mid A_{1}\right\rangle \approx-\left\langle\phi \mid A_{1}^{\prime}\right\rangle,
$$

(equality holds if $P\left\{A_{1}\right\}$ is exactly $1 / 2$ ). Since the reversed picture, conditioned on $A_{1}^{\prime}$, exists, neighbouring high-speed regions in figure 5(a) could be interpreted as equal-magnitude high-speed regions whose strengths have been smeared by other lessdominant large-scale dispersive motions in the averaging process. Thus, a detailed description of physical processes far away from $\boldsymbol{x}_{\times}$is difficult to ascertain. One may be tempted to believe from the conditional average fields that these structures are aligned in the streamwise direction; instantaneous visualizations (see Monty et al. 2007) suggest that these are in fact meandering structures, which would have been obscured in the averaging over the periodic domain in our study. However, this apparent meandering coherence could equally well be interpreted as adjoined regions of shorter coherence, which are individually well captured by the conditional averaging. The spanwise scale appears to be approximately $\delta$.

Figure $5(b)$ shows the spanwise structure of the relationship between $u_{L}$ and $\widetilde{u}_{S}$ : near the wall, $u_{L}$ and $\widetilde{u}_{S}$ are positively correlated, but above $z / \delta \approx 0.1$, they are negatively correlated. This crossing point is different from $z / \delta \approx 0.2$ seen in figure $4(a)$. The discrepancy is resolved by noting the inclusive $\left(A_{1}=u_{L}-U>0\right)$ and non-collocated (two-point) nature of the conditioning used for the averages $\left\langle u_{L} \mid A_{1}\right\rangle$ and $\left\langle\widetilde{u}_{S} \mid A_{1}\right\rangle$, as well as the single plane rather than integral representation. In contrast, the correlation $R$ (see (3.17)) is constructed from the one-point collocated statistic, $\left\langle u_{L} \widetilde{u}_{S}\right\rangle \equiv \int\left\langle u_{L} \mid u_{L}\right\rangle\left\langle\widetilde{u}_{S} \mid u_{L}\right\rangle p\left(u_{L}\right) \mathrm{d} u_{L}$, which is not the same as $\sum_{A}\left\langle u_{L} \mid A\right\rangle\left\langle\widetilde{u}_{S} \mid A\right\rangle P\{A\}$. The opposite $u_{L}-\widetilde{u}_{S}$ configuration of the weaker flanking regions in figure $5(b)$ suggests that these too experience phase reversal.

Figure $5(c, d)$ shows the streamwise-wall-normal structure of the dominant largescale motion and the relationship between the small and large scales. Despite a filter size of $\delta$, the coherence indicated in the figure suggests a wavelength of order $6 \delta$, suggesting that the very long structures are the dominant contributors to the $u_{L}\left(\boldsymbol{x}_{\times}\right)-U<0$ signal. Clearly, the large-scale coherence has a streamwise phase that is dependent on the wall-normal location, at least where the coherence is strongest. Close to the wall this phase variation is weak, while the conditional averages with $\boldsymbol{x}_{\times}^{+}=(0,0,1000) \Leftrightarrow \boldsymbol{x}_{\times} / \delta=(0,0,0.5)$ shown in figure 7 show that far from the wall the phase variation with increasing $z / \delta$ is also weak, but close to $\pi$ rather than zero. In the intermediate region, the phase changes rapidly with wall-normal distance. The contours of constant $\left|\widetilde{u}_{S}^{+}-\left\langle\widetilde{u}_{S}^{+}\right\rangle\right|$in figure $5(d)$ reveal a surface that cuts through the large-scale low-speed region at a diagonal such that the region of negative correlation is larger where $\Delta x<0$ but smaller where $\Delta x>0$. The angle of this separatrix, at least in the aforementioned intermediate region, is about $14^{\circ}$, suggesting that the modulation reversal (Mathis et al. 2009a) is related to the structure inclination angle (Marusic \& Heuer 2007). 


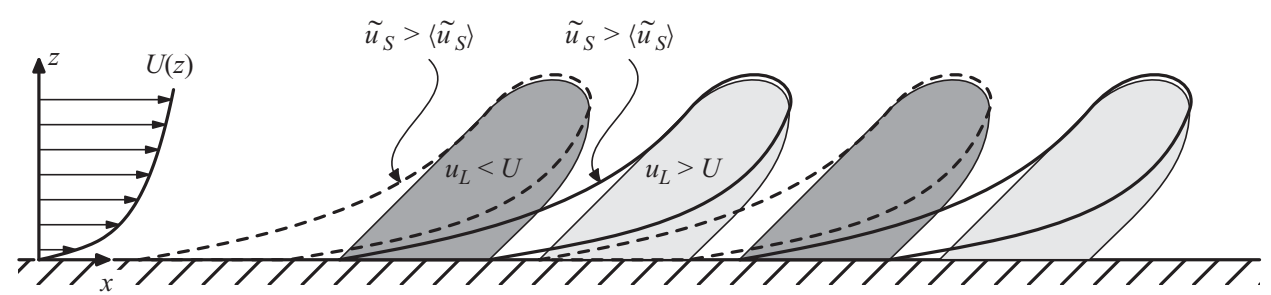

FIGURE 6. Sketch of observed large-scale-small-scale interaction.
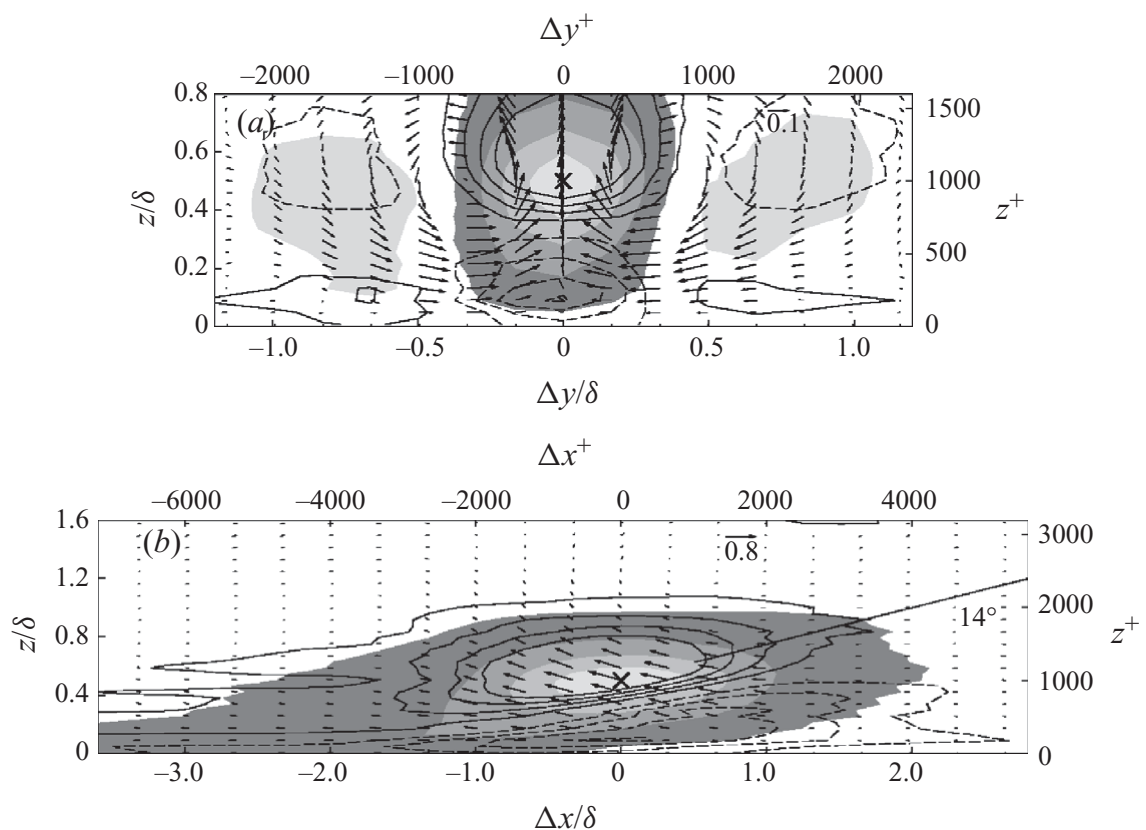

FiguRE 7. Conditionally averaged streamwise velocity fields in $(a)$ spanwise-wall-normal and (b) streamwise-wall-normal planes, with $\left\langle\boldsymbol{u}_{L}^{+} \mid A_{2}\right\rangle(\Delta \boldsymbol{x})$ and $\left\langle\widetilde{u}_{S}^{+} \mid A_{2}\right\rangle(\Delta \boldsymbol{x})$, where $A_{2}$ is the low-speed event $u_{L}\left(\boldsymbol{x}_{\times}\right)-U<0$ with probability $P\left\{A_{2}\right\}=0.47$ at $\boldsymbol{x}_{\times} / \delta=(0,0,0.5)$ : filled contours of $\left|\left\langle u_{L}^{+} \mid A_{2}\right\rangle-U^{+}\right|=0.1,0.3,0.5,0.7$, line contours of $\left|\left\langle\widetilde{u}_{S}^{+} \mid A_{2}\right\rangle-\left\langle\widetilde{u}_{S}^{+}\right\rangle\right|=0.01,0.02$, $0.03,0.04$. Darker to lighter shades and dashed lines for negative values. Vectors represent in-plane velocity components. Data from snapshot of $R e_{\tau}=2 \mathrm{~K}$ channel flow LES, run G1b (table 1).

Figure $5(c, d)$ and $P\left\{A_{1}\right\} \approx 1 / 2$ suggest the stylized picture of a streamwise train of alternating high-speed and low-speed regions with the shape in figure $5(c)$, as illustrated in figure 6 . We propose that this sign change of $u_{L}-U$ determines the shape of the $\widetilde{u}_{S}$ region over a range of wall-normal distances. Consider the governing equation for $\widetilde{u}_{S}^{2}$, which contains the production term $-2 \widetilde{u}_{S}^{2} \partial u_{L} / \partial x$ (Reynolds \& Hussain 1972). Now, $\partial u_{L} / \partial x<0$ between a high-speed region placed upstream $(-\Delta x)$ of a low-speed region. Then, the production $-2 \widetilde{u}_{S}^{2} \partial u_{L} / \partial x>0$ increases $\widetilde{u}_{S}^{2}$, resulting in the picture $5(d)$. The opposite mechanism applies between a low-speed region placed upstream of a high-speed region, in which case $-2 \widetilde{u}_{S}^{2} \partial u_{L} / \partial x<0$, a backscatter of small-scale streamwise energy. This results in the quarter-phase shift between the $u_{L}$ and $\widetilde{u}_{S}$ region. 

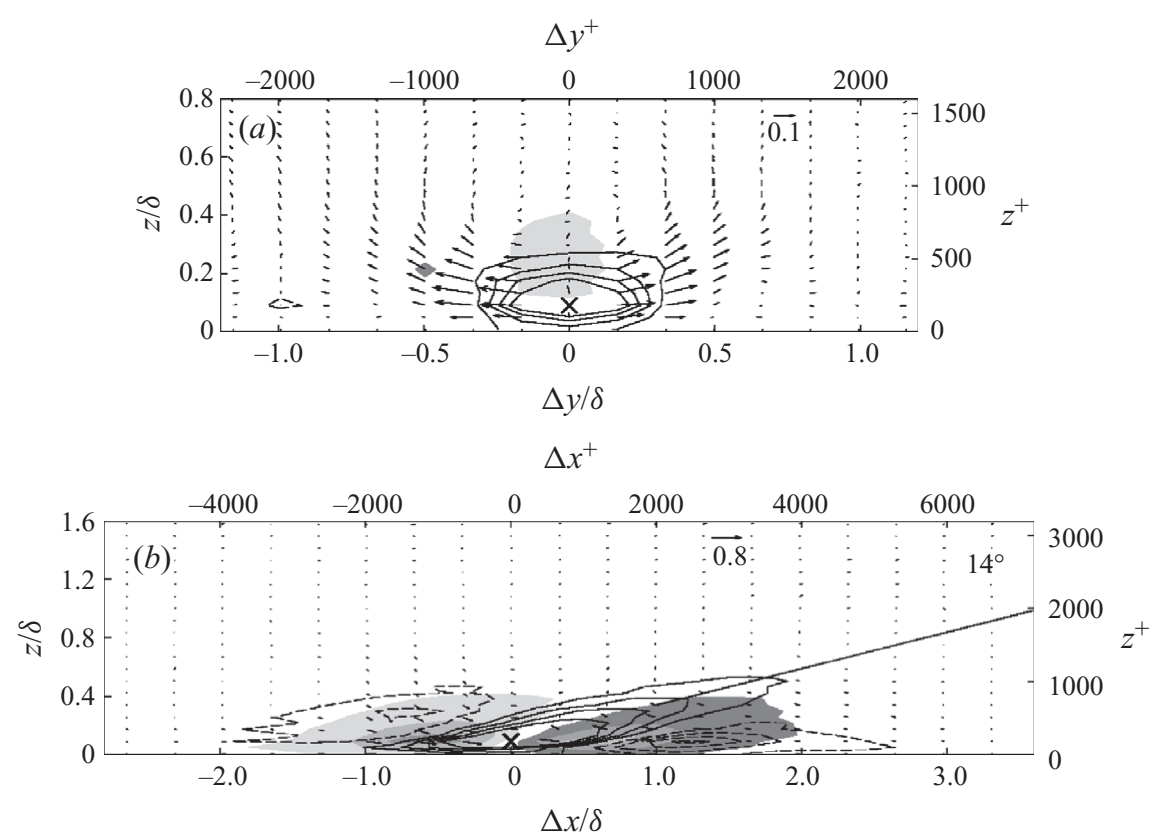

FIGURE 8. Conditionally averaged streamwise velocity fields in (a) spanwise-wall-normal and (b) streamwise-wall-normal planes, with $\left\langle\boldsymbol{u}_{L}^{+} \mid A_{3}\right\rangle(\Delta \boldsymbol{x})$ and $\left\langle\widetilde{u}_{S}^{+} \mid A_{3}\right\rangle(\Delta \boldsymbol{x})$, where $A_{3}$ is the streamwise high-speed-to-low-speed boundary event $\left(\partial u_{L} / \partial x\right)\left(\boldsymbol{x}_{\times}\right)<0$ with probability $P\left\{A_{3}\right\}=0.48$ at $\boldsymbol{x}_{\times} / \delta=(0,0,0.090)$ : filled contours of $\left|\left\langle u_{L}^{+} \mid A_{3}\right\rangle-U^{+}\right|=0.1,0.3,0.5,0.7$, line contours of $\left|\left\langle\widetilde{u}_{S}^{+} \mid A_{3}\right\rangle-\left\langle\widetilde{u}_{S}^{+}\right\rangle\right|=0.01,0.02,0.03,0.04$. Darker to lighter shades and dashed lines for negative values. Vectors represent in-plane velocity components. Data from snapshot of $R e_{\tau}=2 \mathrm{~K}$ channel flow LES, run G1b (table 1).

A clearer picture emerges when we compute averages conditioned on the $u_{L}>0$ to $u_{L}<0$ boundary, signalled by the event $A_{3}=\left(\partial u_{L} / \partial x\right)\left(\boldsymbol{x}_{\times}\right)<0$ (figure 8), interpreted as a quarter-phase streamwise shift of figure $5(c, d)$. As expected, $\widetilde{u}_{S}$ is lowest precisely where $\partial u_{L} / \partial x$ is minimum (at $\boldsymbol{x}_{\times}$), figure $8(b)$. The bulge-like shape of the $u_{L}$ regions is preserved (see figure $8 a$ ), although with smaller sizes. Like the average conditioned on $A_{1}$, this figure is also reversible, with $P\left\{A_{3}\right\}=0.48$.

The interactions further from the wall can be investigated by repeating the conditional averaging at $\boldsymbol{x}_{\times} / \delta=(0,0,0.5)$. A different relationship between the largeand small-scale activity emerges, as shown in figures 7 and 9 . Instead of the 0 $\pi / 2$ phase difference close to the wall, $\left|u_{L}^{+}-U^{+}\right|$and $\left|\widetilde{u}_{S}^{+}-\left\langle\widetilde{u}_{S}^{+}\right\rangle\right|$are substantially out of phase, that is, the phase difference is approximately $\pi$. This variation is in good agreement with boundary-layer results over a Reynolds number range of three decades, namely Bandyopadhyay \& Hussain (1984) and the recent work of Guala et al. (2010). From these other works, it would be expected that the large and small scales have close-to-zero phase difference very close to the wall; the LES formulation prevents us from confirming this point.

From figure 5 we estimate the peak values: $u_{L}^{+} \approx 0.8, v_{L}^{+} \approx 0.2$ and $w_{L}^{+} \approx 0.05$. Thus, the peak shear stress carried directly by these large-scale structures is estimated from this conditionally averaged picture as $-\left\langle u_{L} w_{L}\right\rangle^{+} \approx 0.04$, and the peak largescale streamwise intensity is estimated as $\left\langle u_{L}^{2}\right\rangle^{+} \approx 0.64$. The relative magnitude and phase of the other velocity components associated with the large-scale structure can be confirmed by looking at equivalent conditional averages for the other 

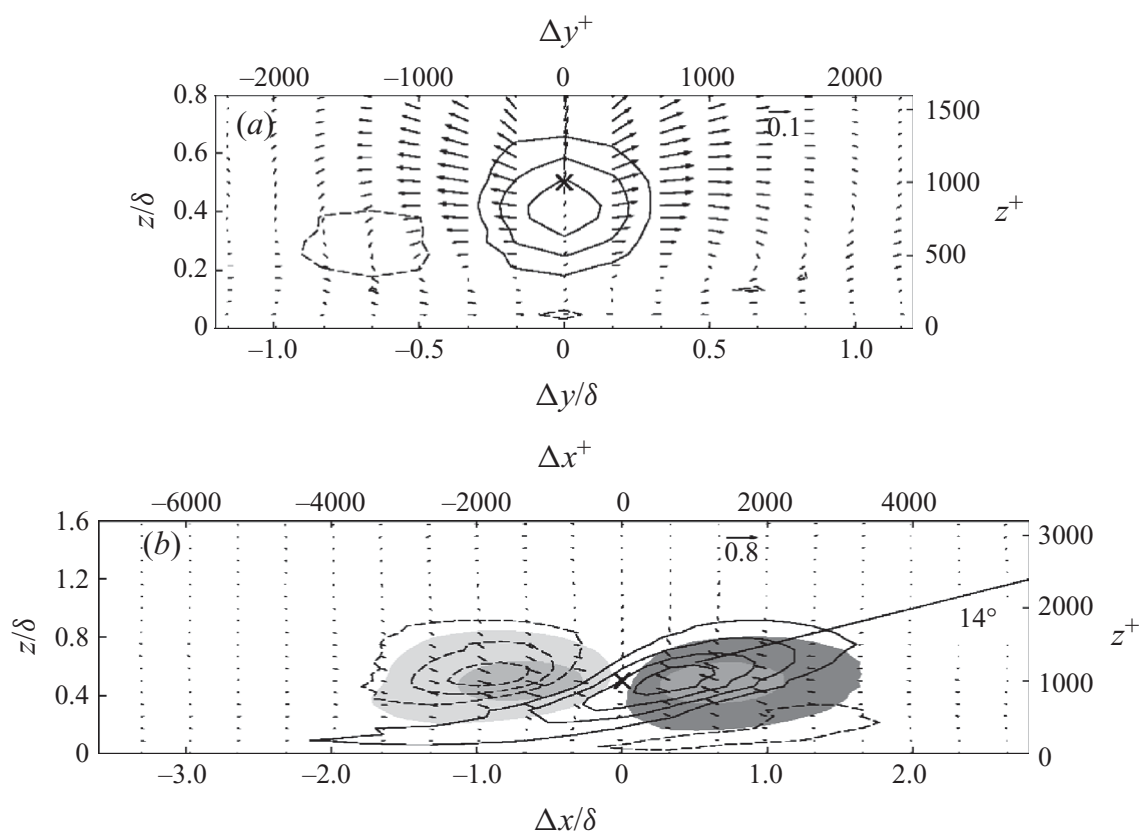

FIGURE 9. Conditionally averaged streamwise velocity fields in $(a)$ spanwise-wall-normal and (b) streamwise-wall-normal planes, with $\left\langle\boldsymbol{u}_{L}^{+} \mid A_{4}\right\rangle(\Delta \boldsymbol{x})$ and $\left\langle\widetilde{u}_{S}^{+} \mid A_{4}\right\rangle(\Delta \boldsymbol{x})$, where $A_{4}$ is the streamwise high-speed-to-low-speed boundary event $\left(\partial u_{L} / \partial x\right)\left(\boldsymbol{x}_{\times}\right)<0$ with probability $P\left\{A_{4}\right\}=0.48$ at $\boldsymbol{x}_{\times} / \delta=(0,0,0.5)$ : filled contours of $\left|\left\langle u_{L}^{+} \mid A_{4}\right\rangle-U^{+}\right|=0.1,0.3,0.5,0.7$, line contours of $\left|\left\langle\widetilde{u}_{S}^{+} \mid A_{4}\right\rangle-\left\langle\widetilde{u}_{S}^{+}\right\rangle\right|=0.01,0.02,0.03,0.04$. Darker to lighter shades and dashed lines for negative values. Vectors represent in-plane velocity components. Data from snapshot of $R e_{\tau}=2 \mathrm{~K}$ channel flow LES, run G1b (table 1).

velocity components. Note that it is extremely difficult to obtain this sort of data experimentally, so we are effectively using the LES data in a predictive capacity to complete the description of the trends in the structure of the large-scale motions.

Figures $10(a, b), 11(a, b)$ and $12(a, b)$ reveal that while the spanwise velocity exhibits a footprint consistent with the implied swirl of figure 5 , such that $v_{L}=0$ statistically on the conditioning plane, but with a wall-normal phase variation similar to that exhibited by the streamwise velocity $u_{L}$. By contrast, the wall-normal velocity velocities in figures $10(c), 11(c)$ and $12(c)$ are substantially in phase in the wall-normal direction, independent of the conditioning criterion.

The conditional averaging technique can be taken one step further to demonstrate that, despite a large wall-normal footprint in the streamwise velocity, the large scales will contribute locally to the mean shear stress because of the relative wall-normal phases of the large-scale streamwise and wall-normal velocities. Figures 10(c), 11(c) and 12(c) show the conditioned shear stress distributions associated with the large scales, that is the product of the conditional averages of $u_{L}$ and $w_{L}$. A conditionally averaged $u_{L} w_{L}$ yields a different answer that includes the effects of smaller structures since $\left\langle u_{L} w_{L} \mid A_{1}\right\rangle \neq\left\langle u_{L} \mid A_{1}\right\rangle\left\langle w_{L} \mid A_{1}\right\rangle$. The relative phases of $u_{L}$ and $w_{L}$ imply that there will be a contribution to the mean shear stress close to the wall. Note that this result is subject to the success of the conditional averaging in capturing streamwise coherence in $u_{L}$ and $w_{L}$, but the trend is believed to be robust. 

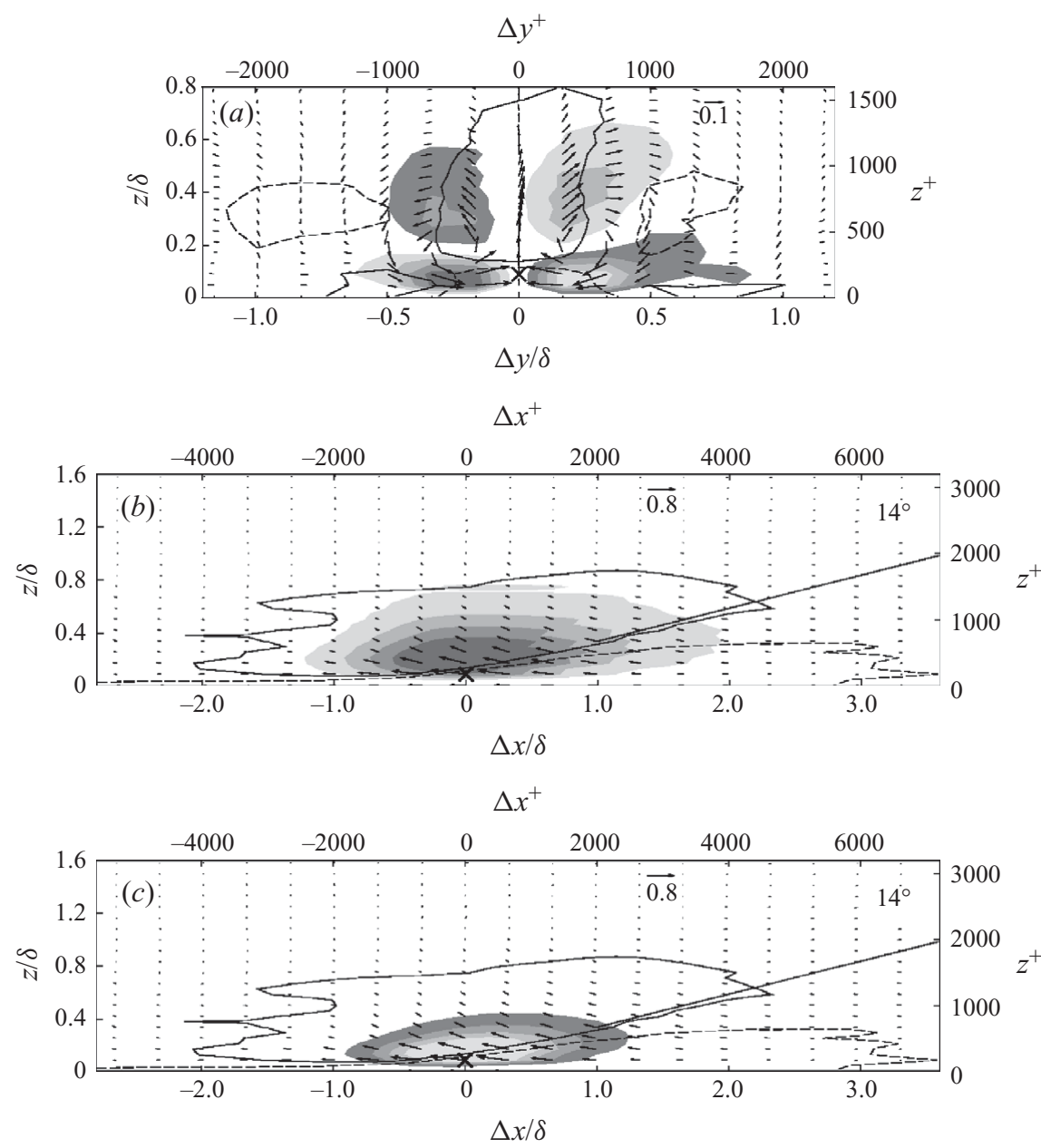

FIGURE 10. Spanwise and wall-normal velocities, and Reynolds stress associated with the conditionally averaged streamwise velocity in $(a)$ spanwise-wall-normal and $(b, c)$ streamwise-wall-normal planes, with $\left\langle\boldsymbol{u}_{L}^{+} \mid A_{1}\right\rangle(\Delta \boldsymbol{x})$ and $\left\langle\widetilde{u}_{S}^{+} \mid A_{1}\right\rangle(\Delta \boldsymbol{x})$, where $A_{1}$ is the low-speed event $u_{L}\left(\boldsymbol{x}_{\times}\right)-U<0$ with probability $P\left\{A_{1}\right\}=0.53$ at $\boldsymbol{x}_{\times} / \delta=(0,0,0.090)$ : (a) filled contours of $\left|\left\langle\widetilde{v}_{L}^{+} \mid A_{1}\right\rangle\right|=0.04,0.08,0.12,0.16$; (b) filled contours of $\left|\left\langle\widetilde{w}_{L}^{+} \mid A_{1}\right\rangle\right|=0.03,0.06,0.09,0.12$; (c) filled contours of $\left|\left\langle\widetilde{w}_{L}^{+} \mid A_{1}\right\rangle\left(\left\langle\widetilde{u}_{L}^{+} \mid A_{1}\right\rangle-U^{+}\right)\right|=0.02,0.04,0.06,0.08$; and $(a-c)$ line contours of $\left|\left\langle\widetilde{u}_{S}^{+} \mid A_{1}\right\rangle-\left\langle\widetilde{u}_{S}^{+}\right\rangle\right|=0.01$. Darker to lighter shades and dashed lines for negative values. Vectors represent in-plane velocity components. Data from snapshot of $R e_{\tau}=2 \mathrm{~K}$ channel flow LES, run G1b (table 1).

Finally, we note that the same large-scale-small-scale interaction is largely unaffected by Reynolds number differences, as shown in the comparison between $R e_{\tau}=2 \mathrm{~K}$ and $R e_{\tau}=200 \mathrm{~K}$ data (figures 13 and 14). This is consistent with the well-known collapse of the streamwise intensity (and spectrum) in outer variables. It is to be expected that any significant changes in the conditionally averaged fields due to the effect of increasing Reynolds number would be observed nearer the wall, exactly in the region that is not accessible in our LES data. 

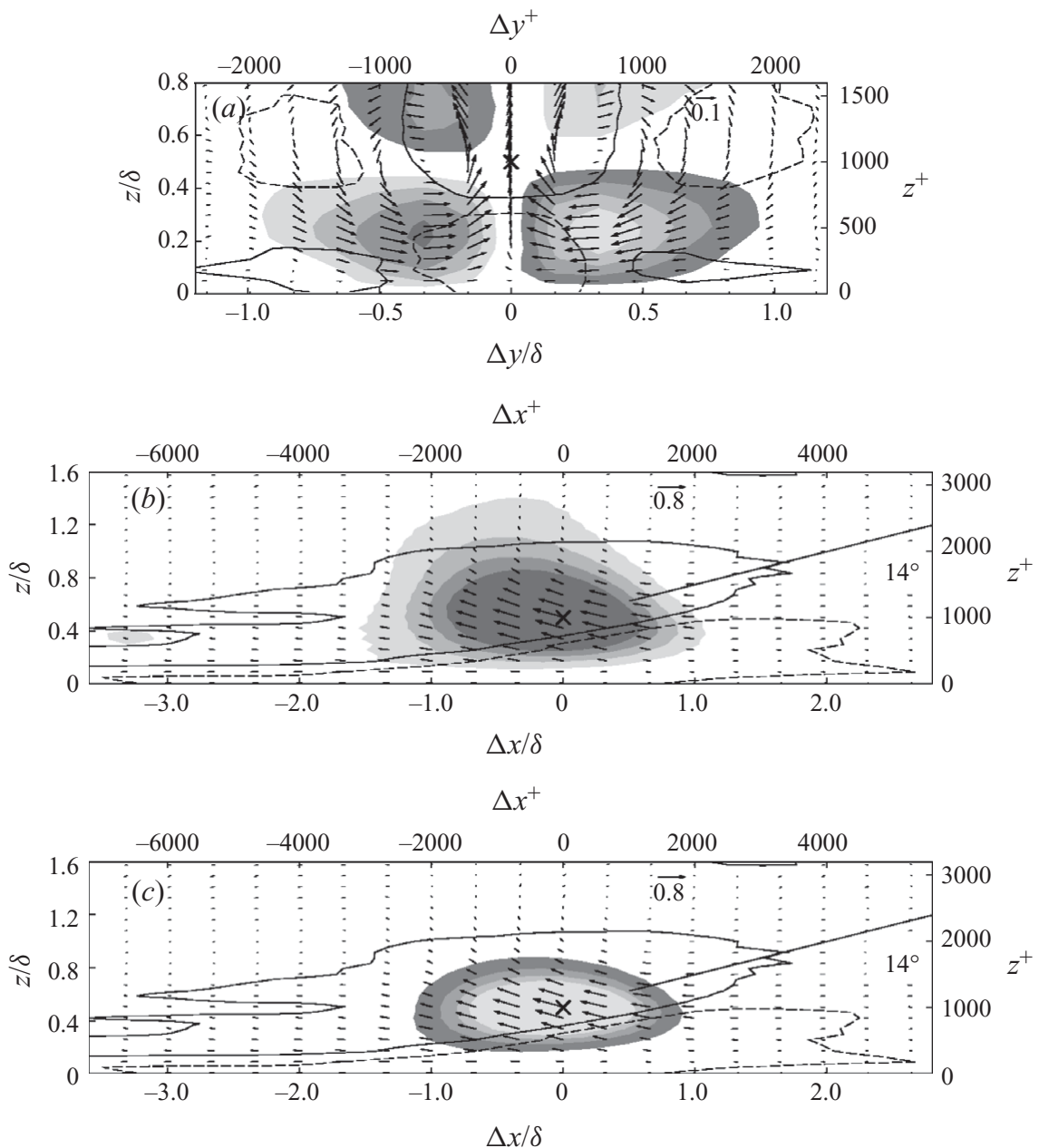

FIGURE 11. Spanwise and wall-normal velocities, and Reynolds stress associated with the conditionally averaged streamwise velocity in $(a)$ spanwise-wall-normal and $(b, c)$ streamwise-wall-normal planes, with $\left\langle\boldsymbol{u}_{L}^{+} \mid A_{2}\right\rangle(\Delta \boldsymbol{x})$ and $\left\langle\widetilde{\boldsymbol{u}}_{S}^{+} \mid A_{2}\right\rangle(\Delta \boldsymbol{x})$, where $A_{2}$ is the low-speed event $u_{L}\left(\boldsymbol{x}_{\times}\right)-U<0$ with probability $P\left\{A_{2}\right\}=0.47$ at $\boldsymbol{x}_{\times} / \delta=(0,0,0.5)$ : (a) filled contours of $\left|\left\langle\widetilde{v}_{L}^{+} \mid A_{1}\right\rangle\right|=0.04,0.08,0.12,0.16 ;(b)$ filled contours of $\left|\left\langle\widetilde{w}_{L}^{+} \mid A_{1}\right\rangle\right|=0.03,0.06,0.09,0.12$; (c) filled contours of $\left|\left\langle\widetilde{w}_{L}^{+} \mid A_{1}\right\rangle\left(\left\langle\widetilde{u}_{L}^{+} \mid A_{1}\right\rangle-U^{+}\right)\right|=0.02,0.04,0.06,0.08$; and $(a-c)$ line contours of $\left|\left\langle\widetilde{u}_{S}^{+} \mid A_{1}\right\rangle-\left\langle\widetilde{u}_{S}^{+}\right\rangle\right|=0.01$. Darker to lighter shades and dashed lines for negative values. Vectors represent in-plane velocity components. Data from snapshot of $R e_{\tau}=2 \mathrm{~K}$ channel flow LES, run G1b (table 1).

\section{Summary and conclusions}

We have designed a series of LES runs that are well suited to the investigation of large-scale structures in a long channel. The observations from this study lend themselves to an interpretation of the ubiquitous influence of large-scale structures in turbulent channel flow, consistent with the boundary-layer experiments of Bandyopadhyay \& Hussain (1984), Mathis et al. (2009a) and Guala et al. (2010).

Using simultaneous time and spatial data to construct the spatio-temporal spectrum, we compute the convection velocity, and show that departure from Taylor's frozenturbulence hypothesis is noticeable near the wall, $z / \delta<0.08$, and for large scales, $>6 \delta$, 

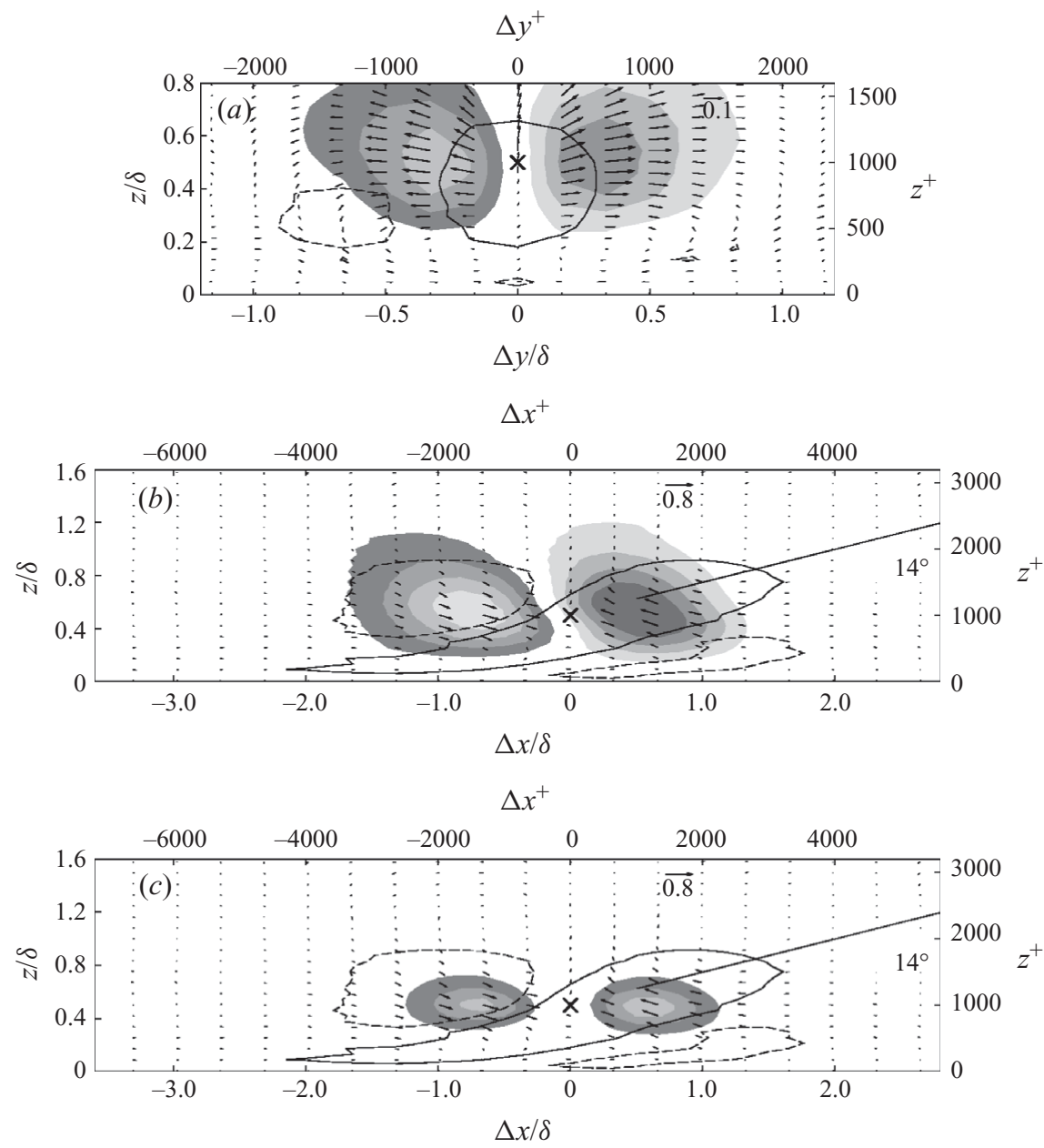

FIGURE 12. Spanwise and wall-normal velocities, and Reynolds stress associated with the conditionally averaged streamwise velocity in $(a)$ spanwise-wall-normal and $(b, c)$ streamwisewall-normal planes, with $\left\langle\boldsymbol{u}_{L}^{+} \mid A_{4}\right\rangle(\Delta \boldsymbol{x})$ and $\left\langle\widetilde{u}_{S}^{+} \mid A_{4}\right\rangle(\Delta \boldsymbol{x})$, where $A_{4}$ is the streamwise high-speed-to-low-speed boundary event $\left(\partial u_{L} / \partial x\right)\left(\boldsymbol{x}_{\times}\right)<0$ with probability $P\left\{A_{4}\right\}=0.48$ at $\boldsymbol{x}_{\times} / \delta=(0,0,0.5):$ (a) filled contours of $\left|\left\langle\widetilde{v}_{L}^{+} \mid A_{1}\right\rangle\right|=0.04,0.08,0.12,0.16$; (b) filled contours of $\left|\left\langle\widetilde{w}_{L}^{+} \mid A_{1}\right\rangle\right|=0.03,0.06,0.09,0.12 ; \quad(c)$ filled contours of $\left|\left\langle\widetilde{w}_{L}^{+} \mid A_{1}\right\rangle\left(\left\langle\widetilde{u}_{L}^{+} \mid A_{1}\right\rangle-U^{+}\right)\right|=0.02,0.04,0.06,0.08 ;$ and $(a-c)$ line contours of $\mid\left\langle\widetilde{u}_{S}^{+} \mid A_{1}\right\rangle-$ $\left\langle\widetilde{u}_{S}^{+}\right\rangle \mid=0.01$. Darker to lighter shades and dashed lines for negative values. Vectors represent in-plane velocity components. Data from snapshot of $R e_{\tau}=2 \mathrm{~K}$ channel flow LES, run G1b (table 1).

in channel flow at $R e_{\tau}=2 \mathrm{~K}$. This is consistent with the footprint of the very large scales reaching down to the wall. The opposite effect - that the large scales convect slower than the local mean - is also observed for these large scales away from the wall $(z / \delta=0.5)$. This, too, is consistent with the conditional averages that show that these large-scale structures reach far away from the wall.

Flow fields constructed from conditional averages confirm the extent of the influence of scales with $\lambda_{x} \approx 6 \delta$ and reveal the wall-normal dependence of the spatial relationship between the high-speed large-scale region and the underlying small-scale intensity. The LES results appear to underline that the apparent amplitude modulation effect 

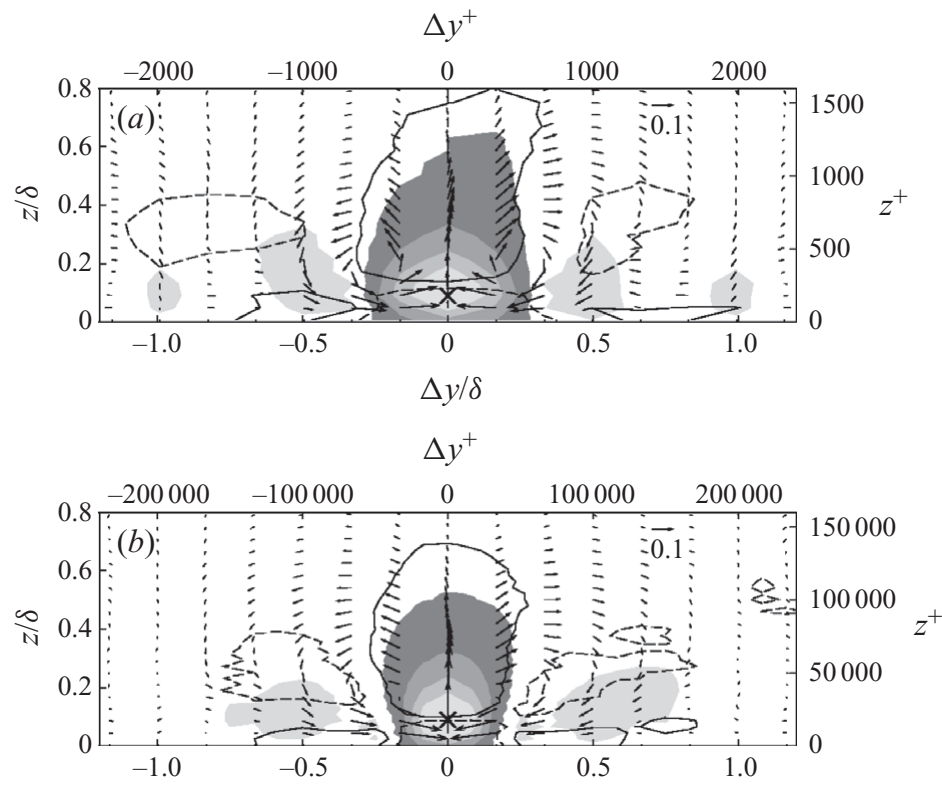

$\Delta y / \delta$
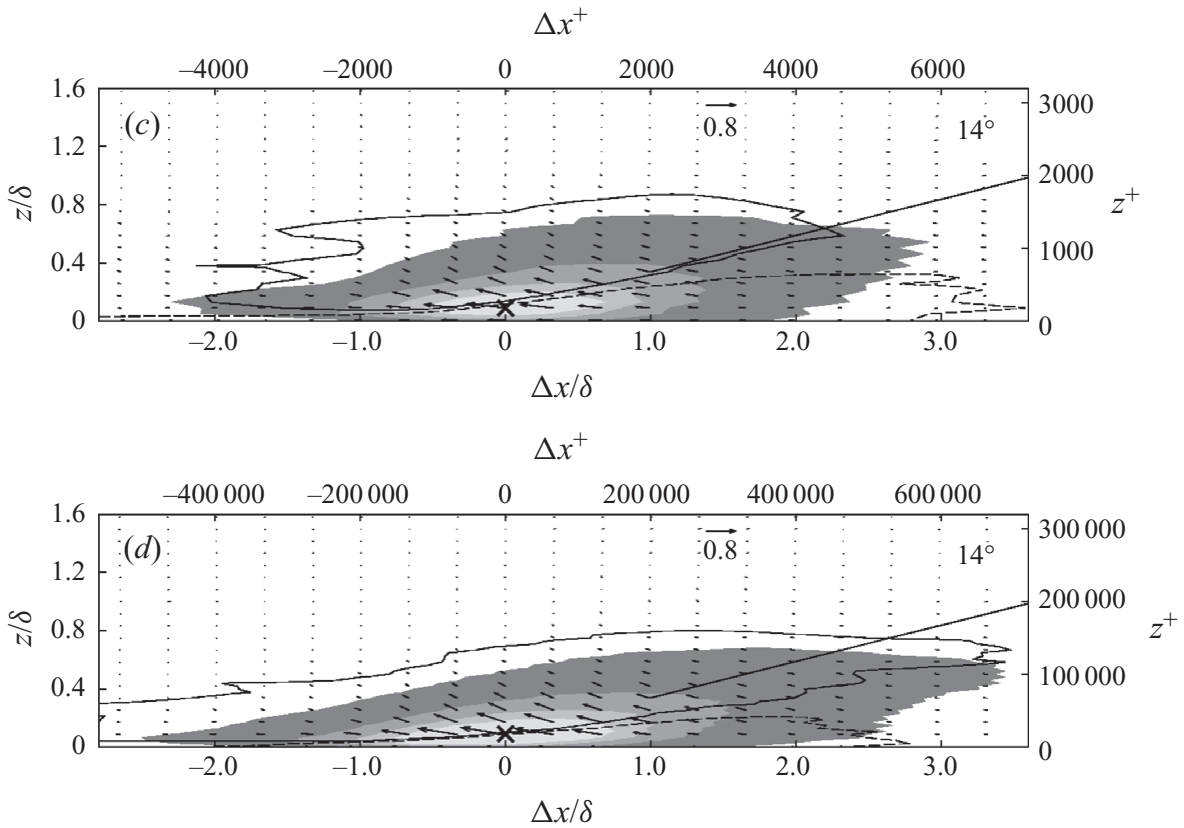

FIGURE 13. Reynolds number effect, $(a, c) R e_{\tau}=2 \mathrm{~K},(b, d) R e_{\tau}=200 \mathrm{~K}$, on conditionally averaged streamwise velocity fields in the $(a, b)$ spanwise-wall-normal and $(c, d)$ streamwisewall-normal planes, with $\left\langle\boldsymbol{u}_{L}^{+} \mid A_{1}\right\rangle(\Delta \boldsymbol{x})$ and $\left\langle\widetilde{u}_{S}^{+} \mid A_{1}\right\rangle(\Delta \boldsymbol{x})$, where $A_{1}$ is the low-speed event $u_{L}\left(\boldsymbol{x}_{\times}\right)-U<0$ with probability $P\left\{A_{1}\right\}=0.53\left(R e_{\tau}=2 \mathrm{~K}\right)$ and $P\left\{A_{1}\right\}=0.51 \quad\left(R e_{\tau}=200 \mathrm{~K}\right)$ at $\boldsymbol{x}_{\times} / \delta=(0,0,0.090)$ : filled contours of $\left|\left\langle u_{L}^{+} \mid A_{1}\right\rangle-U^{+}\right|=0.1,0.3,0.5,0.7$, line contour of $\left|\left\langle\widetilde{u}_{S}^{+} \mid A_{1}\right\rangle-\left\langle\widetilde{u}_{S}^{+}\right\rangle\right|=0.01$. Darker to lighter shades and dashed lines for negative values. Vectors represent in-plane velocity components. Data from snapshots of $R e_{\tau}=2 \mathrm{~K}$ and $R e_{\tau}=200 \mathrm{~K}$ channel flow LES, respectively runs G1b and H3 (table 1). 

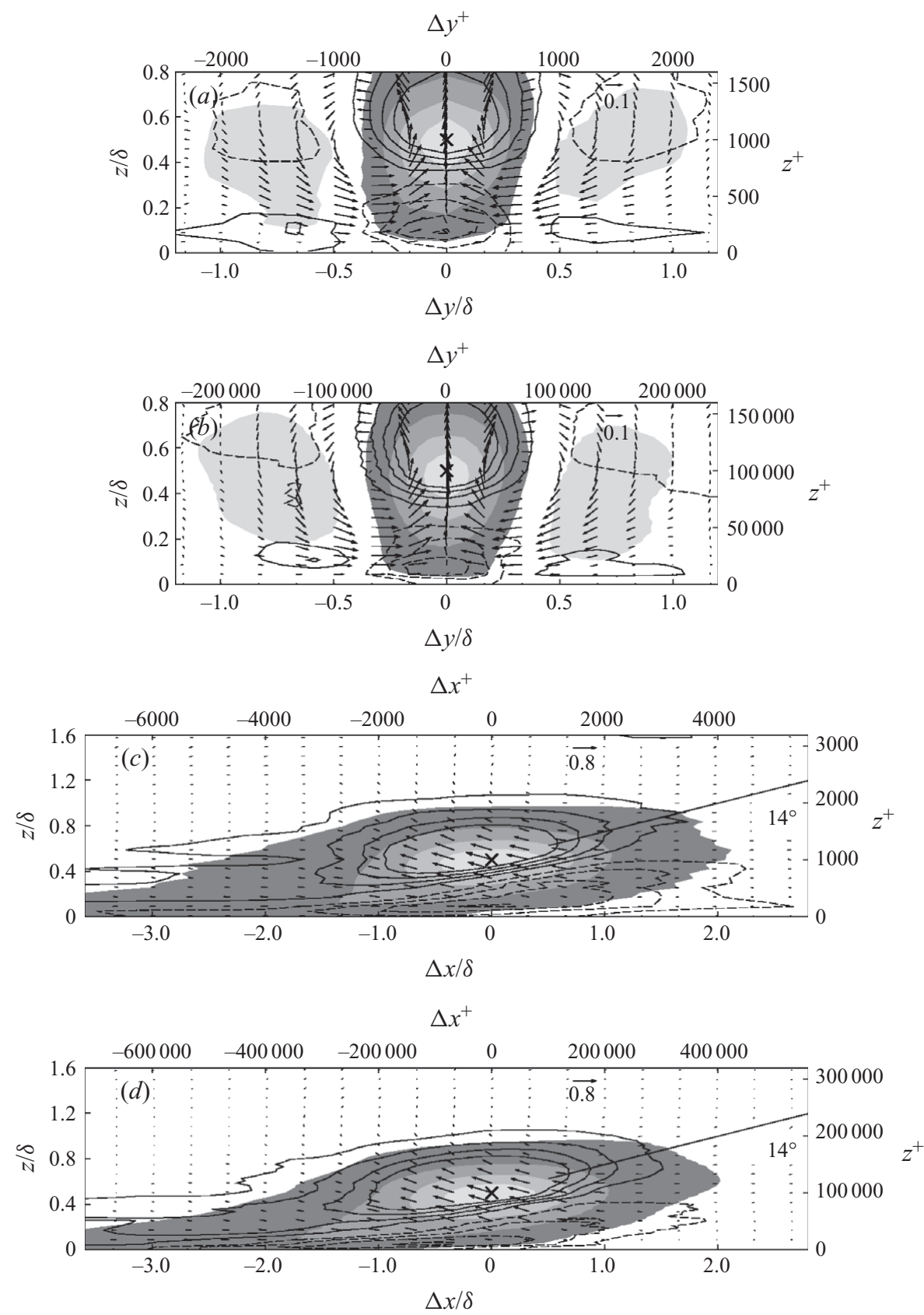

FIGURE 14. Reynolds number effect, $(a, c) R e_{\tau}=2 \mathrm{~K},(b, d) R e_{\tau}=200 \mathrm{~K}$, on conditionally averaged streamwise velocity fields in $(a, b)$ spanwise-wall-normal and $(c, d)$ streamwisewall-normal planes, with $\left\langle\boldsymbol{u}_{L}^{+} \mid A_{2}\right\rangle(\Delta \boldsymbol{x})$ and $\left\langle\widetilde{u}_{S}^{+} \mid A_{2}\right\rangle(\Delta \boldsymbol{x})$, where $A_{2}$ is the low-speed event $u_{L}\left(\boldsymbol{x}_{\times}\right)-U<0$ with probability $P\left\{A_{2}\right\}=0.47\left(R e_{\tau}=2 \mathrm{~K}\right)$ and $P\left\{A_{2}\right\}=0.47\left(R e_{\tau}=200 \mathrm{~K}\right)$ at $\boldsymbol{x}_{\times} / \delta=(0,0,0.5)$ : filled contours of $\left|\left\langle u_{L}^{+} \mid A_{1}\right\rangle-U^{+}\right|=0.1,0.3,0.5,0.7$, line contours of $\left|\left\langle\widetilde{u}_{S}^{+} \mid A_{1}\right\rangle-\left\langle\widetilde{u}_{S}^{+}\right\rangle\right|=0.01,0.02,0.03,0.04$. Darker to lighter shades and dashed lines for negative values. Vectors represent in-plane velocity components. Data from snapshots of $R e_{\tau}=2 \mathrm{~K}$ and $R e_{\tau}=200 \mathrm{~K}$ channel flow LES, respectively runs G1b and H3 (table 1). 
is better described in terms of the spatial phase between the large and the small scales; indeed our correlation coefficients, $R_{\rho}$ and $R_{\tau}$, are formulated in terms of this phase relationship. In this context, the zero in the correlation between the largeand small-scale activity described in $\$ 3.2$ can be interpreted as the location where, on average, the corresponding signals are $\pi / 2$ out of phase. A picture emerges in which the small scales are nominally in phase with the large scales near the wall and $\pi$ out of phase further from the wall. In the intervening region, where the phase difference is approximately $\pi / 2$, the small scales track the sign of $\partial u_{L} / \partial x$, as per the small-scale production/backscatter term $-2 \widetilde{u}_{S}^{2} \partial u_{L} / \partial x$. We can interpret the small-scale activity, $\widetilde{u}_{S}$, in terms of local structure, noting that the inferred locus of the maximum small-scale energy corresponds to the well-known structure inclination angle of approximately $10^{\circ}-20^{\circ}$, at least at this Reynolds number.

We conclude by observing that the very large scales appear to dictate some of the turbulence behaviour close to the wall, with interesting implications for the 'top-down versus bottom-up' debate concerning the dynamical significance of different regions of the flow. It is perhaps more correct to say simply that the two regions can now be determined to be inextricably linked.

The authors acknowledge the support of the NSF under grants CBET-0651754 (DC) and CAREER-0747672 (BJM). It is our pleasure to acknowledge Professor Dale Pullin's generous support for this investigation, and useful discussions with Dr Ati Sharma and Dr Michele Guala.

\section{REFERENCES}

Del Álamo, J. C. \& JimÉnez, J. 2009 Estimation of turbulent convection velocities and corrections to Taylor's approximation. J. Fluid Mech. 640, 5-26.

del Álamo, J. C., Jiménez, J., Zandonade, P. \& Moser, R. D. 2004 Scaling of the energy spectra of turbulent channels. J. Fluid Mech. 500, 135-144.

Balakumar, B. J. \& Adrian, R. J. 2007 Large- and very-large-scale motions in channel and boundary-layer flows. Phil. Trans. R. Soc. A 365, 665-681.

Bandyopadhyay, P. R. \& Hussain, A. K. M. F. 1984 The coupling between scales in shear flows. Phys. Fluids 27, 2221-2228.

Cantwell, B. J. 1981 Organized motion in turbulent flow. Annu. Rev. Fluid Mech. 13, 457-515.

Chapman, D. R. 1979 Computational aerodynamics, development and outlook. AIAA J. 17, 12931313.

Chung, D. \& Pullin, D. I. 2009 Large-eddy simulation and wall modelling of turbulent channel flow. J. Fluid Mech. 631, 281-309.

Dennis, D. J. C. \& Nickels, T. B. 2008 On the limitations of Taylor's hypothesis in constructing long structures in a turbulent boundary layer. J. Fluid Mech. 614, 197-206.

Favre, A. J., Gaviglio, J. J. \& Dumas, R. 1967 Structure of velocity space-time correlations in a boundary layer. Phys. Fluids 10, S138-145.

Guala, M., Hommema, S. E. \& Adrian, R. J. 2006 Large-scale and very-large-scale motions in turbulent pipe flow. J. Fluid Mech. 554, 521-542.

Guala, M., Metzger, M. J. \& McKeon, B. J. 2010 Interactions within the turbulent boundary layer at high Reynolds number. J. Fluid Mech. (to appear).

HoYAS, S. \& JimÉNEZ, J. 2006 Scaling of the velocity fluctuations in a turbulent channels up to $R e_{\tau}=2003$. Phys. Fluids 18, 011702 .

Hutchins, N. \& Marusic, I. $2007 a$ Evidence of very long meandering features in the logarithmic region of turbulent boundary layers. J. Fluid Mech. 579, 1-28.

Hutchins, N. \& Marusic, I. $2007 b$ Large-scale influences in near-wall turbulence. Phil. Trans. R. Soc. A 365, 647-664.

Jovanović, M. R. \& Bamieh, B. 2005 Componentwise energy amplification in channel flows. J. Fluid Mech. 534, 145-183. 
Kim, K. C. \& Adrian, R. J. 1999 Very large-scale motion in the outer layer. Phys. Fluids 11, $417-422$.

Kovasznay, L. S. G., Kibens, V. \& Blackwelder, R. F. 1970 Large-scale motion in the intermittent region of a turbulent boundary layer. J. Fluid Mech. 41, 283-325.

Krogstad, P.-Å., Kaspersen, J. H. \& Rimestad, S. 1998 Convection velocities in a turbulent boundary layer. Phys. Fluids 10, 949-957.

Marusic, I. \& HeuER, W. D. C. 2007 Reynolds number invariance of the structure inclination angle in wall turbulence. Phys. Rev. Lett. 99, 114504.

Mathis, R., Hutchins, N. \& Marusic, I. 2009a Large-scale amplitude modulation of the small-scale structures in turbulent boundary layers. J. Fluid Mech. 628, 311-337.

Mathis, R., Monty, J. P., Hutchins, N. \& Marusic, I. $2009 b$ Comparison of large-scale amplitude modulation in turbulent boundary layers, pipes, and channel flows. Phys. Fluids 21, 111703.

McKeon, B. J. \& Sharma, A. S. 2010 A critical layer model for turbulent pipe flow. J. Fluid Mech. (to appear).

MisRa, A. \& Pullin, D. I. 1997 A vortex-based subgrid stress model for large-eddy simulation. Phys. Fluids 9, 2443-2454.

Monty, J. P. \& ChOng, M. S. 2009 Turbulent channel flow: comparison of streamwise velocity data fom experiments and direct numerical simulation. J. Fluid Mech. 633, 461-474.

Monty, J. P., Hutchins, N., Ng, H. C. H., Marusic, I. \& Chong, M. S. 2009 A comparison of turbulent pipe, channel and boundary layer flows. J. Fluid Mech. 632, 431-442.

Monty, J. P., Stewart, J. A., Williams, R. C. \& Chong, M. S. 2007 Large-scale features in turbulent pipe and channel flows. J. Fluid Mech. 589, 147-156.

Morrison, J. F., McKeon, B. J., Jiang, W. \& Smits, A. J. 2004 Scaling of the streamwise velocity component in turbulent pipe flow. J. Fluid Mech. 508, 99-131.

RaO, K. N., Narasimha, R. \& Narayanan, M. A. B. 1971 The 'bursting' phenomenon in a turbulent boundary layer. J. Fluid Mech. 48, 339-352.

Reynolds, W. C. \& Hussain, A. K. M. F. 1972 The mechanics of an organized wave in turbulent shear flow. Part 3. Theoretical models and comparisons with experiments. J. Fluid Mech. 54, 263-288.

Voelkl, T., Pullin, D. I. \& Chan, D. C. 2000 A physical-space version of the stretched-vortex subgrid-stress model for large-eddy simulation. Phys. Fluids 12, 1810-1825.

WiLls, J. A. B. 1964 On convection velocities in turbulent shear flows. J. Fluid Mech. 20, 417-432. 